\title{
Donaldson theory on non-Kählerian surfaces and class VII surfaces with $b_{2}=1$
}

\author{
Andrei Teleman
}

May 1, 2005

\section{Contents}

1 Introduction

1.1 The main result . . . . . . . . . . . . . . . . 2

1.2 Donaldson Theory revisited . . . . . . . . . . . . . 3

1.3 The strategy of the proof $\ldots \ldots \ldots \ldots$

2 Background material 7

2.1 The Picard group, the Gauduchon degree and the square roots of $[\mathcal{O}] \ldots \ldots \ldots \ldots \ldots \ldots \ldots$

3 A moduli space of simple bundles 8

3.1 Classifying simple filtrable bundles . . . . . . . . . . . . 8

3.2 Topological properties . . . . . . . . . . . . . 15

4 A moduli space of stable bundles $\quad \mathbf{1 6}$

4.1 Classifying filtrable stable bundles . . . . . . . . . . . . 16

4.2 A collar around the reductions . . . . . . . . . . 18

4.3 The missing centers . . . . . . . . . . . . . . 22

4.4 A smooth compact complex curve in the moduli space . . . . . . 24

5 Families of bundles parameterized by a curve 25

\begin{abstract}
We prove that any class VII surface with $b_{2}=1$ has curves. This implies the "Global Spherical Shell conjecture" in the case $b_{2}=1$ :

Any minimal class VII surface with $b_{2}=1$ admits a global spherical shell, hence it is isomorphic to one of the surfaces in the known list.

By the results in [LYZ, Te1, which treat the case $b_{2}=0$ and give complete proofs of Bogomolov's theorem, one has a complete classification of all class $V I I$-surfaces with $b_{2} \in\{0,1\}$.
\end{abstract}


The main idea of the proof is to show that a certain moduli space of $P U(2)$-instantons on a surface $X$ with no curves (if such a surface existed) would contain a closed Riemann surface $Y$ whose general points correspond to non-filtrable holomorphic bundles on $X$. Then we pass from a family of bundles on $X$ parameterized by $Y$ to a family of bundles on $Y$ parameterized by $X$, and we use the algebraicity of $Y$ to obtain a contradiction.

The proof uses essentially techniques from Donaldson theory: compactness theorems for moduli spaces of $P U(2)$-instantons and the Kobayashi-Hitchin correspondence on surfaces.

\section{Introduction}

\subsection{The main result}

A class VII surface is a compact complex surface $X$ with $b_{1}(X)=1$ and $\operatorname{kod}(X)=-\infty$. The topological invariants of such a surface are

$$
c_{2}(X)=-c_{1}(X)^{2}=b_{2}(X), b_{+}^{2}(X)=0 .
$$

Therefore the intersection form $H^{2}(X, \mathbb{Z}) /$ Tors $\times H^{2}(X, \mathbb{Z}) /$ Tors $\rightarrow \mathbb{Z}$ is negative definite so, by Donaldson's first theorem, it is trivial over $\mathbb{Z}$.

Class VII surfaces are not classified yet. This is probably the most important gap in the Enriques-Kodaira classification table. The case $b_{2}=0$ is completely understood:

Theorem 1.1 Every class VII-surface with $b_{2}=0$ is isomorphic to either a Hopf surface or an Inoue surface.

This result was stated by Bogomolov a long time ago [Bo1, Bo2, but his proof is long and difficult to follow (see BHPV] p. 230); complete proofs appeared in [Te1] and [LYZ.

Both proofs are based on the Kobayashi-Hitchin correspondence on nonKählerian surfaces (see [Bu1, [LY], [LT1]) applied to a single holomorphic bundle: the tangent bundle of the surface.

The main result of this paper is:

Theorem 1.2 Let $X$ be a class VII surface with $b_{2}(X)=1$. Then $X$ has an effective divisor $C>0$ with

$$
c_{1}^{\mathbb{Q}}(\mathcal{O}(C)) \in\left\{ \pm c_{1}^{\mathbb{Q}}\left(\mathcal{K}_{X}\right), 0,2 c_{1}^{\mathbb{Q}}\left(\mathcal{K}_{X}\right)\right\},
$$

where $c_{1}^{\mathbb{Q}}$ stands for the first Chern class in rational cohomology.

Using Theorem 11.2 in $[\mathrm{Na}$, one concludes that

Corollary 1.3 Any minimal class VII surface $X$ with $b_{2}(X)=1$ possesses a spherical shell, hence it belongs to the known class of surfaces. 
The proof of the main theorem is again based on the Kobayashi-Hitchin correspondence but, unlike Theorem 1.1 - which uses the correspondence for a single bundle - it requires a careful examination of the geometry of a certain moduli space of instantons (stable bundles) on $X$; therefore it is much closer in spirit to techniques used in Donaldson theory. In particular, one needs essentially the Kobayashi-Hitchin correspondence as an isomorphism of moduli spaces, and the compactness theorems for moduli spaces of $P U(2)$ instantons (which, in the non-Kählerian framework, cannot be obtained by complex geometric methods).

We mention that many arguments can be partially generalized for class $V I I_{0}$ surfaces with higher $b_{2}$ (see [Te2]). By a result of Dloussky-Oeljeklaus-Toma DOT, any class $V I I_{0}$ surface which has $b_{2}$ rational curves, contains a global spherical shell, hence it belongs to the known class. Therefore, the classification problem for class VII surfaces reduces to the existence of "sufficiently many" curves.

\subsection{Donaldson Theory revisited}

We explain now the gauge theoretical tools needed in the proof ([Do, [DK], [LT1]):

Let $(M, g)$ be a compact oriented Riemannian 4-manifold and $E$ be a Hermitian 2-bundle on $M$. For a connection $A$ on $E$, denote as usually by $F_{A}$ its curvature and by $F_{A}^{0}$ its trace-free part.

Put $L:=\operatorname{det}(E)$. We fix a Hermitian connection $a \in \mathcal{A}(L)$ and denote by $\mathcal{A}_{a}(E)$ the space of Hermitian connections on $E$ which induce $a$ on $L$. Such connections are called sometimes "oriented connections". $\mathcal{A}_{a}(E)$ is an affine space over the vector space $A^{1}(s u(E))$; the gauge group $S U(E)$ of unitary automorphisms of determinant 1 acts on this affine space naturally. The moduli space of projectively ASD connections in $\mathcal{A}_{a}(E)$ is

$$
\mathcal{M}_{a}^{\mathrm{ASD}}(E):=\left\{A \in \mathcal{A}_{a}(E) \mid\left(F_{A}^{0}\right)^{+}=0\right\} / S U(E) \subset \mathcal{A}_{a}(E) / S U(E)=: \mathcal{B}_{a}(E)
$$

Let $P$ be the principal unitary frame bundle of $E$ and $\bar{P}:=P / S^{1}$ the associated $P U(2)$-bundle. One has a natural identification $\mathcal{A}_{a}(E) \simeq \mathcal{A}(\bar{P})$ which yields a surjection

$$
\mathcal{M}_{a}^{\mathrm{ASD}}(E) \longrightarrow \mathcal{M}^{\mathrm{ASD}}(\bar{P})
$$

with finite fibers. This surjection is an isomorphism in the simply connected case, but in general is not! The point is that the gauge group $S U(E)$ could be slightly smaller than the automorphism group $\operatorname{Aut}(\bar{P})$. This phenomenon can be easily understood as follows (see [LT1, p. 141-145 for details):

An element $\rho \in H^{1}\left(X, \mathbb{Z}_{2}\right)$ can be interpreted as a flat $S^{1}$-connection $a_{\rho}$ on a Hermitian line bundle $L_{\rho}$, which comes with a tautological unitary isomorphism $L_{\rho}^{\otimes 2}=X \times \mathbb{C}$. The bundles $E$ and $E \otimes L_{\rho}$ have the same determinant line bundle and are isomorphic; more precisely, there exists a unitary isomorphism 
$f: E \rightarrow E \otimes L_{\rho}$ with $\operatorname{det}(f) \equiv 1$. The map $A \mapsto f^{-1}\left(A \otimes a_{\rho}\right)$ descends to a well defined map $\otimes \rho: \mathcal{B}_{a}(E) \rightarrow \mathcal{B}_{a}(E)$. In this way one obtains an $H^{1}\left(X, \mathbb{Z}_{2}\right)$ action on the quotients $\mathcal{B}_{a}(E), \mathcal{M}_{a}^{\mathrm{ASD}}(E)$, and $\mathcal{M}^{\mathrm{ASD}}(\bar{P})$ is just the $H^{1}\left(X, \mathbb{Z}_{2}\right)$ quotient of $\mathcal{M}_{a}^{\mathrm{ASD}}(E)$.

Note that the irreducible part $\left[\mathcal{M}_{a}^{\mathrm{ASD}}(E)\right]^{\text {irr }}$ of $\mathcal{M}_{a}^{\mathrm{ASD}}(E)$ can contain fixed points of this action; in other words there exist in general irreducible unitary connections which project on reducible $P U(2)$-connections.

In classical gauge theory, one usually works with the simpler moduli space $\mathcal{M}^{\mathrm{ASD}}(\bar{P})$ and ignores $\mathcal{M}_{a}^{\mathrm{ASD}}(E)$, because both spaces should carry equivalent differential topological information.

For our proof it is important to consider $\mathcal{M}_{a}^{\mathrm{ASD}}(E)$ (rather than $\mathcal{M}^{\mathrm{ASD}}(\bar{P})$ ), because the $H^{1}\left(X, \mathbb{Z}_{2}\right)$-symmetry of this space plays a crucial role in the proof.

The precise form of the Kobayashi-Hitchin correspondence we need is the following (see [Bu1, [LT1, [LT2], [LY]):

Theorem 1.4 Let $(X, g)$ be a compact complex surface endowed with a Gauduchon metric $G$ and $E$ a Hermitian bundle on $X$. Fix a holomorphic structure $\mathcal{L}$ on the Hermitian line bundle $L:=\operatorname{det}(E)$ and let $a \in \mathcal{A}(L)$ be the corresponding Chern connection. There is a natural real analytic isomorphism

$$
\left[\mathcal{M}_{a}^{\mathrm{ASD}}(E)\right]^{\mathrm{irr}} \stackrel{K H \simeq}{\longrightarrow} \mathcal{M}_{g}^{\text {st }}(E, \mathcal{L})
$$

Here $\mathcal{M}_{g}^{\text {st }}(E, \mathcal{L})$ denotes the moduli space of $g$-stable holomorphic structures on $E$ which induce $\mathcal{L}$ on $L=\operatorname{det} E$, modulo the complex gauge group $\operatorname{SL}(E)$ (see [LT1, [LT2]).

When $h$ is chosen such that $\operatorname{det}(h)$ is a Hermitian-Einstein metric on $\mathcal{L}$, this statement follows formally from the standard Kobayashi-Hitchin correspondence between irreducible Hermitian-Einstein connections and stable bundles [LT1, LY. The general statement is a very special case of the universal KobayashiHitchin correspondence for oriented pairs [LT2, but it can be easily deduced from the case when $\operatorname{det}(h)$ is Hermitian-Einstein, by noting that the left hand moduli space is in fact independent of $a$, up to canonical isomorphism.

Denote by $\lambda$ the semiconnection on $L$ given by the Dolbeault operator of $\mathcal{L}$. Let $\mathcal{A}^{0,1}(E)$ be the complex affine space of semiconnections (" $(0,1)$ connections") on $E$ (see [Do, [LO], LT1]) and $\mathcal{A}_{\lambda}^{0,1}(E)$ the subspace of semiconnections which induce $\lambda$ on $L$.

The Kobayashi-Hitchin isomorphism $K H$ is induced by the affine map

$$
\mathcal{A}_{a} \rightarrow \mathcal{A}_{\mathcal{L}}^{0,1}(E), A \mapsto \bar{\partial}_{A}
$$

Using a standard corollary to Uhlenbeck's compactness theorem DK, one gets the following important result, which cannot be obtained by complex geometric methods.

Corollary 1.5 In the conditions of Theorem 1.4 suppose that $\operatorname{rk}(E)=2$, and $4 c_{2}(E)-c_{1}(E)^{2} \leq 3$. Then the complex moduli space $\mathcal{M}^{\mathrm{st}}(E, \mathcal{L})$ can be identified 
with an open set of the compact moduli space $\mathcal{M}_{a}^{\mathrm{ASD}}(E)$. Therefore, in this case, $\mathcal{M}^{\text {st }}(E, \mathcal{L})$ can be compactified by adding only the reducible part of $\mathcal{M}_{a}^{\mathrm{ASD}}(E)$, which can be identified with the set of split polystable bundles $\mathcal{M} \oplus\left[\mathcal{L} \otimes \mathcal{M}^{-1}\right]$, $2 \operatorname{deg}_{g}(\mathcal{M})=\operatorname{deg}_{g}(\mathcal{L}), c_{1}(\mathcal{M})\left(c_{1}(\mathcal{L})-c_{1}(\mathcal{M})\right)=c_{2}(E)$.

The compactness of $\mathcal{M}_{a}^{\mathrm{ASD}}(E)$ follows from the fact that every $P U(2)$-instanton has non-positive Pontrjagin number so, under the assumption $p_{1}(\bar{P}) \geq-3$, the lower strata of the Uhlenbeck compactification of $\mathcal{M}^{\mathrm{ASD}}(\bar{P})$ are empty.

Remark: Suppose that we are in the conditions of the above corollary. When $b_{1}(X)$ is odd, the compactification $\mathcal{M}_{a}^{\mathrm{ASD}}(E)$ of $\mathcal{M}^{\text {st }}(E, \mathcal{L})$ is not in general a complex space.

For instance one can get a moduli space of stable bundles isomorphic to an open disk $D \subset \mathbb{C}$, which is compactified in the natural way by adding a circle. The point is that the stratum of reducible connections in $\mathcal{M}_{a}^{\mathrm{ASD}}(E)$ (split polystable bundles with fixed determinant) can have odd real dimension.

This remark also shows that, in the non-Kählerian framework, one cannot hope to find a purely complex geometric way to compactify the moduli spaces of stable bundles (as the Gieseker compactification in the algebraic case).

The Kobayashi-Hitchin correspondence (Theorem 1.4) can be regarded as a practical method for computing moduli spaces of projectively ASD connections with complex geometric methods. Note however that the classification of holomorphic bundles on a non-algebraic manifold is in general a very difficult problem. Indeed, on a non-algebraic manifold there exists in general non-filtrable bundles (see section 3.1). Such bundles are always stable with respect to any Gauduchon metric. On the other hand there exists no general construction or classification method for non-filtrable bundles $[\mathrm{BLP}$.

\subsection{The strategy of the proof}

The idea of the proof is the following:

Let $X$ be a class VII surface with $b_{2}(X)=1$. We fix a Gauduchon metric $g$ on $X$ such that $\operatorname{deg}_{g}\left(\mathcal{K}_{X}\right) \neq 0$ (which is possible since $c_{1}\left(\mathcal{K}_{X}\right)^{2} \neq 0$ ), and consider the moduli space $\mathcal{M}^{\text {st }}:=\mathcal{M}_{g}^{\text {st }}\left(0, \mathcal{K}_{X}\right)$ of $g$-stable bundles $\mathcal{E}$ with $\operatorname{det}(\mathcal{E}) \simeq \mathcal{K}_{X}$ and $c_{2}=0$. The expected complex dimension of this space is 1 .

Put

$$
p:=\left|\operatorname{Tors}\left(H^{2}(X, \mathbb{Z})\right)\right|, q:=\left|\operatorname{Tors}_{2}\left(H^{2}(X, \mathbb{Z})\right)\right|,
$$

where Tors 2 stands for the 2 -torsion of an abelian group. One has $0<q \leq p$. Supposing that $X$ has no curves $C$ with

$$
c_{1}^{\mathbb{Q}}(\mathcal{O}(C)) \in\left\{ \pm c_{1}^{\mathbb{Q}}\left(\mathcal{K}_{X}\right), 0,2 c_{1}^{\mathbb{Q}}\left(\mathcal{K}_{X}\right)\right\},
$$


we show that the compactification $\overline{\mathcal{M}^{\text {st }}}$ of $\mathcal{M}^{\text {st }}$ given by Corollary 1.5 contains as an open set the disjoint union

$$
\left[\coprod_{i=1}^{p-q} D_{i}\right] \coprod\left[\coprod_{j=1}^{q} P_{j}\right]
$$

where $D_{i}$ are closed disks, and $P_{j}$ are copies of one of the two spaces illustrated below

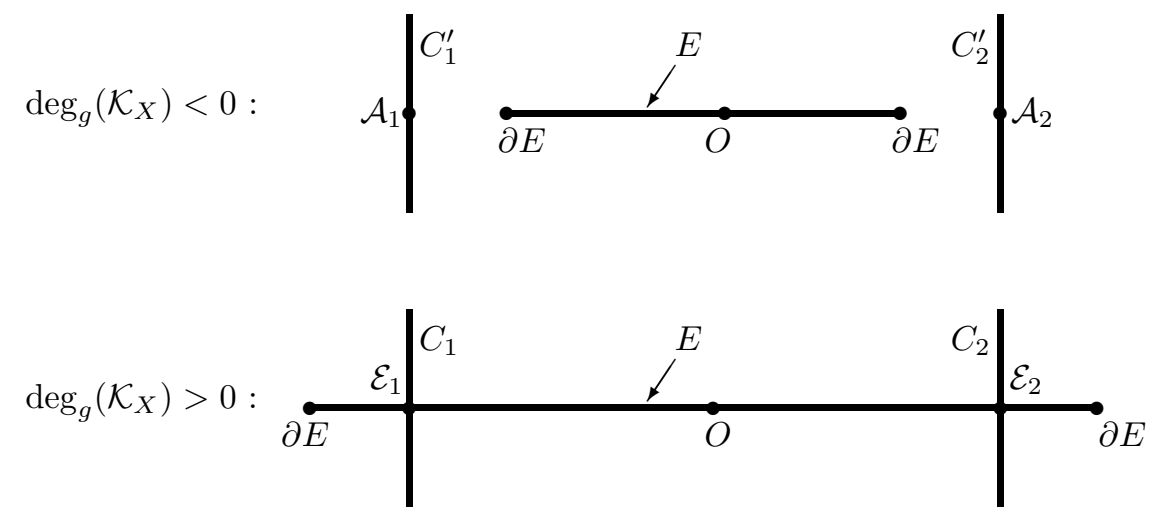

One must choose the first picture when $\operatorname{deg}_{g}\left(\mathcal{K}_{X}\right)<0$ and the second when $\operatorname{deg}_{g}\left(\mathcal{K}_{X}\right)>0$. In both pictures, the segment $E$ represents a closed disk with center $O$. The points of $\stackrel{\circ}{E} \backslash\{O\}$ correspond to filtrable stable bundles, which can be completely classified. The origin $O$ is a non-filtrable bundle which is obtained as pushforward of a holomorphic line bundle on a bicovering; it is a fixed point under a natural $\mathbb{Z}_{2}$-action. The circle $\partial E$ is the stratum of reducible solutions (split polystable bundles) mentioned in Corollary 1.5. Moreover, the locus of reducible solutions is the union of the boundaries $\partial E_{j} \subset P_{j}$ and the boundaries $\partial D_{i}$ (hence it is a disjoint union of $p$ circles).

Each vertical segment $C_{i}^{\prime}$ (respectively $C_{i}$ ) represents a small open disk whose only filtrable point is the center $\mathcal{A}_{i}$ (respectively $\mathcal{E}_{i}$ ). The $\mathcal{E}_{i}$ 's are the only singularities of the moduli space in the second case, whereas $\mathcal{M}^{\text {st }}$ is smooth in the first case.

The main idea is the following: since $\overline{\mathcal{M}^{\text {st }}}$ is compact, it follows that the disks $C_{i}^{\prime}\left(C_{i}\right)$ are part of a compact complex subspace of the moduli space. One can easily see that this compact subspace is in fact a smooth (possibly non-connected) closed Riemann surface.

The crucial point here is that our disjoint union is embedded injectively as an open set in the moduli space, so that the vertical and the horizontal loci belong to different irreducible components.

The presence in the moduli space of a smooth closed complex curve $Y$ which contains both filtrable and non-filtrable points leads to a contradiction (see section (5). The first step is to put together the bundles $\left(\mathcal{E}_{y}\right)_{y \in Y}$ and get a 
bundle $\mathcal{E}$ on $Y \times X$. Since $H^{2}\left(Y, \mathcal{O}_{Y}^{*}\right)=0$, there is no obstruction to the construction of such a classifying family $\mathcal{E}$. In particular, one has a family $\left(\mathcal{E}^{x}\right)_{x \in X}$ of bundles on the curve $Y$ parameterized by $X$. We explain briefly, in two simple particular situations, why such a family cannot exist: First, when the bundles $\mathcal{E}^{x}$ are all semistable, one would get a morphism $\varepsilon$ from $X$ into a moduli space of semistable bundles over $Y$, which is an algebraic geometric object. Since our surface has algebraic dimension 0 , it follows that only the case $\varepsilon=$ constant (which gives a very simple family) must be eliminated.

The opposite case is when the $\left(\mathcal{E}^{x}\right)_{x \in X}$ are non-semistable for all $x \in X$. A non semistable 2-bundle $\mathcal{F}$ over a curve has a unique maximal destabilizing sub-line bundle $\mathcal{D}(\mathcal{F})$, which depends "meromorphically" on $\mathcal{F}$. Since the components of $\operatorname{Pic}(Y)$ are also projective manifolds, one has only to discuss the case when the map $x \mapsto\left[\mathcal{D}\left(\mathcal{E}^{x}\right)\right]$ is constant. Putting together the maximal destabilizing line bundles $\mathcal{D}\left(\mathcal{E}^{x}\right)$ we get a subsheaf of rank 1 of $\mathcal{E}$, contradicting the fact that $\mathcal{E}_{y}$ is non-filtrable for generic $y \in Y$.

\section{Background material}

\subsection{The Picard group, the Gauduchon degree and the square roots of $[\mathcal{O}]$}

Let $X$ be a surface, and $\operatorname{Pic}(X)=H^{1}\left(X, \mathcal{O}^{*}\right)$ its Picard group. For a cohomology class $c \in N S(X)$, we will denote by $\operatorname{Pic}^{c}(X)$ the corresponding connected component of $\operatorname{Pic}(X) \cdot \operatorname{Pic}^{0}(X)$ is just the identity component of the group $\operatorname{Pic}(X)$. Let $\operatorname{Pic}^{T}(X)$ be the subgroup of line bundles with torsion Chern class. Therefore

$$
\operatorname{Pic}(X) / \operatorname{Pic}^{0}(X) \simeq N S(X), \operatorname{Pic}(X) / \operatorname{Pic}^{T}(X) \simeq N S(X) / \operatorname{Tors}(N S(X)) .
$$

Let $g$ be a Gauduchon metric $[\mathrm{G}$ ] on $X$, i.e. a Hermitian metric such that $\partial \bar{\partial} \omega_{g}=0$. The degree map associated with $g$ is a group morphism

$$
\operatorname{deg}_{g}: \operatorname{Pic}(X) \longrightarrow \mathbb{R}
$$

defined by

$$
\operatorname{deg}([\mathcal{L}]):=\int_{X} c_{1}(\mathcal{L}, h) \wedge \omega_{g},
$$

where $c_{1}(\mathcal{L}, h)$ denotes the Chern form of any Hermitian metric on $\mathcal{L}$. The degree map is a topological invariant (i.e. it vanishes on $\left.\operatorname{Pic}^{T}(X)\right)$ if and only if $b_{1}(X)$ is even (see [LT1). The restriction $\left.\operatorname{deg}_{g}\right|_{\operatorname{Pic}^{T}(X)}$ is independent of $g$ up to positive multiplicative constant ([LT1, p. 41). Its kernel

$$
\operatorname{Pic}^{f}(X):=\operatorname{ker}\left(\left.\operatorname{deg}_{g}\right|_{\operatorname{Pic}^{T}(X)}: \operatorname{Pic}^{T}(X) \rightarrow \mathbb{R}\right) \subset \operatorname{Pic}^{T}(X)
$$

is always compact, because the Kobayashi-Hitchin correspondence gives an isomorphism of real Lie groups

$$
\operatorname{Hom}\left(\pi_{1}(X), S^{1}\right) \simeq \operatorname{Pic}^{f}(X)
$$


(see [LT1). In the case of surfaces with even $b_{1}$ one has $\operatorname{Pic}^{f}(X)=\operatorname{Pic}^{T}(X)$, whereas for surfaces with odd $b_{1}, \operatorname{Pic}^{f}(X)$ is a real codimension 1 compact subgroup of $\operatorname{Pic}^{T}(X)$.

Let $X$ be an arbitrary class $V I I$ surface. For such a surface the identityconnected component $\operatorname{Pic}^{0}(X)$ of $\operatorname{Pic}(X)$ is isomorphic to $\mathbb{C}^{*}$. More precisely, the natural morphisms

$$
H^{1}\left(X, \mathbb{C}^{*}\right) \longrightarrow \operatorname{Pic}^{T}(X), H^{1}(X, \mathbb{C}) / H^{1}(X, \mathbb{Z}) \longrightarrow \operatorname{Pic}^{0}(X)
$$

are isomorphisms. Therefore, any holomorphic line bundle $\mathcal{L}$ with torsion Chern class has a unique flat connection, and this flat connection is Hermitian if and only if the degree of $\mathcal{L}$ (with respect to any Gauduchon metric) vanishes.

An important role in this article will be played by the square roots of the class $[\mathcal{O}]$ of the trivial holomorphic line bundle. Using (2), one obtains an isomorphism

$$
H^{1}\left(X, \mathbb{Z}_{2}\right)=\operatorname{Hom}\left(\pi_{1}(X), \mathbb{Z}_{2}\right) \simeq \operatorname{Tors}_{2}(\operatorname{Pic}(X))
$$

The long exact sequence associated with $0 \rightarrow \mathbb{Z} \stackrel{2 \cdot}{\longrightarrow} \mathbb{Z} \rightarrow \mathbb{Z}_{2} \rightarrow 0$ gives

$$
0 \longrightarrow H^{1}(X, \mathbb{Z}) / 2 H^{1}(X, \mathbb{Z}) \longrightarrow H^{1}\left(X, \mathbb{Z}_{2}\right) \longrightarrow \operatorname{Tors}_{2}\left(H^{2}(X, \mathbb{Z})\right) \longrightarrow 0
$$

where the epimorphism on the right coincides with the Chern class morphism via the isomorphism (3), and the quotient on the left can be identified with $\mathbb{Z}_{2}$ in our case (because $b_{1}(X)=1$ ).

Therefore, for class VII surfaces one has:

Remark 2.1 A connected component $\operatorname{Pic}^{c}(X)$ of $\operatorname{Pic}(X)$ contains square roots of $[\mathcal{O}]$ if and only if $c \in \operatorname{Tors}_{2}\left(H^{2}(X, \mathbb{Z})\right)$; in this case it contains two square roots which are conjugate under the natural action of $\mathbb{Z}_{2}$.

\section{A moduli space of simple bundles}

\subsection{Classifying simple filtrable bundles}

Let $X$ be a class $V I I$ surface with $b_{2}=1$. We will use the following simplified notations:

$$
\mathcal{K}:=\mathcal{K}_{X}, \operatorname{Pic}:=\operatorname{Pic}(X), \operatorname{Pic}^{c}:=\operatorname{Pic}^{c}(X), \operatorname{Pic}^{T}:=\operatorname{Pic}^{T}(X) .
$$

Let $\mathcal{M}^{s}$ be the moduli space of simple holomorphic rank 2 bundles $\mathcal{E}$ with $\operatorname{det} \mathcal{E} \simeq \mathcal{K}$ and $c_{2}=0$.

We recall that a rank 2 bundle $\mathcal{E}$ on a complex surface $S$ is called filtrable if one of the following equivalent conditions is satisfied:

1. $\mathcal{E}$ has a subsheaf of rank 1 . 
2. There exists a holomorphic line bundle $\mathcal{L}$ on $S$ such that $H^{0}\left(\mathcal{L}^{\vee} \otimes \mathcal{E}\right) \neq 0$.

3. There exist line bundles $\mathcal{L}^{\prime}$ and $\mathcal{L}^{\prime \prime}$ on $S$, a dimension 0 locally complete intersection $Z \subset S$ and a short exact sequence of the form

$$
0 \longrightarrow \mathcal{L}^{\prime} \longrightarrow \mathcal{E} \longrightarrow \mathcal{L}^{\prime \prime} \otimes \mathcal{I}_{Z} \longrightarrow 0 \text {. }
$$

Lemma 3.1 Let $X$ be a class VII surface with $b_{2}=1$. Then $c_{1}(\mathcal{K})$ modulo torsion is a $\mathbb{Z}$-generator of the rank one $\mathbb{Z}$-module $H^{2}(X, \mathbb{Z}) /$ Tors.

This follows from the well-known formula:

$$
c_{1}(\mathcal{K})^{2}=c_{1}(X)^{2}=-b_{2}(X),
$$

for a class $V I I$-surface $X$.

Proposition 3.2 Let $\mathcal{E}$ be a filtrable holomorphic rank 2 bundle on $X$ with $c_{2}(\mathcal{E})=0, c_{1}^{\mathbb{Q}}(\mathcal{E})=c_{1}^{\mathbb{Q}}(\mathcal{K})$. Then there exist holomorphic line bundles $\mathcal{L}, \mathcal{M}$ on $X$ such that

1. $c_{1}^{\mathbb{Q}}(\mathcal{L})=0$.

2. $c_{1}^{\mathbb{Q}}(\mathcal{M})=c_{1}^{\mathbb{Q}}(\mathcal{K})$.

3. $\mathcal{E}$ is either an extension of $\mathcal{L}$ by $\mathcal{M}$ or an extension of $\mathcal{M}$ by $\mathcal{L}$.

Proof: Choose an exact sequence of the form

$$
0 \longrightarrow \mathcal{L}^{\prime} \longrightarrow \mathcal{E} \longrightarrow \mathcal{L}^{\prime \prime} \otimes \mathcal{I}_{Z} \longrightarrow 0 \text {. }
$$

as above. By Lemma 3.1, one can write $c_{1}^{\mathbb{Q}}\left(\mathcal{L}^{\prime}\right)=n c_{1}^{\mathbb{Q}}(\mathcal{K})$, with $n \in \mathbb{Z}$.

$$
c_{2}(\mathcal{E})=0=|Z|+c_{1}^{\mathbb{Q}}\left(\mathcal{L}^{\prime}\right) \cup c_{1}^{\mathbb{Q}}\left(\mathcal{L}^{\prime \prime}\right)=|Z|+n(n-1),
$$

which can only hold when $|Z|=0$, and $n \in\{0,1\}$.

Proposition 3.3 Suppose that $X$ has no effective divisor $C>0$ with

$$
c_{1}^{\mathbb{Q}}(\mathcal{O}(C)) \in\left\{ \pm c_{1}^{\mathbb{Q}}(\mathcal{K}), 0,2 c_{1}^{\mathbb{Q}}(\mathcal{K})\right\} .
$$

1. For every line bundle $\mathcal{L}$ with torsion Chern class there exists a unique (up to isomorphism) rank two bundle $\mathcal{E}_{\mathcal{L}}$ which is the central term of a nontrivial extension

$$
0 \longrightarrow \mathcal{L} \longrightarrow \mathcal{E}_{\mathcal{L}} \longrightarrow \mathcal{K} \otimes \mathcal{L}^{\vee} \longrightarrow 0
$$

For every square root $\mathcal{R}$ of $\mathcal{O}$ there exists a unique (up to isomorphism) rank two bundle $\mathcal{A}_{\mathcal{R}}$ which is the central term of a nontrivial extension

$$
0 \longrightarrow \mathcal{R} \otimes \mathcal{K} \longrightarrow \mathcal{A}_{\mathcal{R}} \longrightarrow \mathcal{R} \longrightarrow 0
$$


2. The bundles $\mathcal{E}_{\mathcal{L}}, \mathcal{A}_{\mathcal{R}}$ are simple. Moreover, $\mathcal{E}_{\mathcal{L}^{\prime}} \neq \mathcal{E}_{\mathcal{L}^{\prime \prime}}$ when $\mathcal{L}^{\prime} \nsucceq \mathcal{L}^{\prime \prime}$ and $\mathcal{A}_{\mathcal{R}^{\prime}} \not \mathcal{A}_{\mathcal{R}^{\prime \prime}}$ when $\mathcal{R}^{\prime} \not \mathcal{R}^{\prime \prime}$.

3. $\mathcal{A}_{\mathcal{R}} \neq \mathcal{E}_{\mathcal{L}}$, for every $[\mathcal{L}] \in \operatorname{Pic}^{T}$ and square root $\mathcal{R}$ of $\mathcal{O}$.

4. $\left[\mathcal{E}_{\mathcal{L}}\right]$ is a smooth point of $\mathcal{M}^{\mathrm{s}}$, except when $[\mathcal{L}] \in \operatorname{Tors}_{2}(\mathrm{Pic})$.

5. For every $[\mathcal{R}] \in \operatorname{Tors}_{2}(\mathrm{Pic}), \mathcal{M}^{\mathrm{s}}$ is reducible (hence singular) at $\left[\mathcal{E}_{\mathcal{R}}\right]$; in a neighborhood of this point $\mathcal{M}^{\mathrm{s}}$ consists of two smooth curves $C_{\mathcal{R}}$ and $\Phi_{\mathcal{R}}$ intersecting transversally at $\left[\mathcal{E}_{\mathcal{R}}\right] . \Phi_{\mathcal{R}}$ is just a neighborhood of $\left[\mathcal{E}_{\mathcal{R}}\right]$ in the 1-parameter family $\left\{\mathcal{E}_{\mathcal{L}} \mid[\mathcal{L}] \in \operatorname{Pic}^{T}\right\}$.

6. For any $[\mathcal{R}] \in \operatorname{Tors}_{2}(\mathrm{Pic})$, the moduli space $\mathcal{M}^{\mathrm{s}}$ is smooth at $\left[\mathcal{A}_{\mathcal{R}}\right]$.

7. The map

$$
\mathfrak{F}: \operatorname{Pic}^{T} \coprod \operatorname{Tors}_{2}(\mathrm{Pic}) \longrightarrow \mathcal{M}^{\mathrm{s}}
$$

given by $\mathcal{L} \mapsto\left[\mathcal{E}_{\mathcal{L}}\right], \mathcal{R} \mapsto\left[\mathcal{A}_{\mathcal{R}}\right]$ parameterizes bijectively the filtrable part of $\mathcal{M}^{\mathrm{s}}$.

Proof: An effective divisor $C \subset X$ with $c_{1}^{\mathbb{Q}}(\mathcal{O}(C))=n c_{1}^{\mathbb{Q}}(\mathcal{K})$ will be called numerically $n$-canonical.

1. By Riemann-Roch Theorem, one has

$$
\chi\left(\mathcal{K}^{\vee} \otimes \mathcal{L}^{\otimes 2}\right)=-1
$$

and $H^{0}\left(\mathcal{K}^{\vee} \otimes \mathcal{L}^{\otimes 2}\right)=H^{2}\left(\mathcal{K}^{\vee} \otimes \mathcal{L}^{\otimes 2}\right)=0$, if $X$ has no numerically anticanonical (respectively numerically bicanonical) curves. Therefore

$$
\operatorname{Ext}^{1}\left(\mathcal{K} \otimes \mathcal{L}^{\vee}, \mathcal{L}\right)=H^{1}\left(\mathcal{K}^{\vee} \otimes \mathcal{L}^{\otimes 2}\right) \simeq \mathbb{C} .
$$

Therefore, up to $\mathbb{C}^{*}$-equivalence, one has a unique nontrivial extension of $\mathcal{K} \otimes \mathcal{L}^{\vee}$ by $\mathcal{L}$. For the second type of extensions, note that $H^{1}(\mathcal{K}) \simeq H^{1}(\mathcal{O})^{\vee} \simeq \mathbb{C}$.

2. A morphism $\varphi: \mathcal{E}_{\mathcal{L}^{\prime}} \rightarrow \mathcal{E}_{\mathcal{L}^{\prime \prime}}$ defines a diagram

$$
\begin{aligned}
& 0 \quad \longrightarrow \quad \mathcal{L}^{\prime} \quad \stackrel{\alpha^{\prime}}{\longrightarrow} \quad \mathcal{E}_{\mathcal{L}^{\prime}} \stackrel{\stackrel{\beta^{\prime}}{\longrightarrow}}{\longrightarrow} \quad \mathcal{L}^{\prime \vee} \otimes \mathcal{K} \quad \longrightarrow 0
\end{aligned}
$$

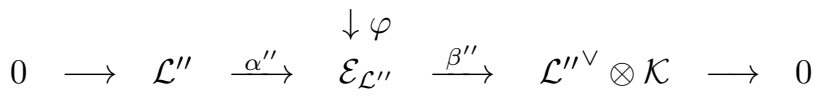

The composition $\beta^{\prime \prime} \circ \varphi \circ \alpha^{\prime}$ vanishes because $X$ has no numerically canonical curves. Therefore, $\beta^{\prime \prime} \circ \varphi$ induces a morphism $q_{\varphi}: \mathcal{L}^{\prime \vee} \otimes \mathcal{K} \rightarrow \mathcal{L}^{\prime \prime} \otimes \mathcal{K}$.

Case a. $\mathcal{L}^{\prime} \nsucceq \mathcal{L}^{\prime \prime}$

In this case $q_{\varphi}$ vanishes because $X$ has no numerically trivial curves. This shows that $\varphi$ factorizes as $\varphi=\alpha^{\prime \prime} \circ \psi$, for a morphism $\psi: \mathcal{E}_{\mathcal{L}^{\prime}} \rightarrow \mathcal{L}^{\prime \prime}$, so $\varphi$ cannot be an isomorphism. Therefore $\mathcal{E}_{\mathcal{L}^{\prime}} \nsucceq \mathcal{E}_{\mathcal{L}^{\prime \prime}}$. 
Case b. $\mathcal{L}^{\prime}=\mathcal{L}^{\prime \prime}$

In this case $q_{\varphi}$ has the form $q_{\varphi}=\zeta \mathrm{id}_{\mathcal{L}^{\prime} \vee \otimes \mathcal{K}}$, so that $\varphi_{\zeta}:=\varphi-\zeta \mathrm{id}_{\mathcal{E}_{\mathcal{L}^{\prime}}}$ factorizes as $\varphi_{\zeta}=\alpha^{\prime \prime} \circ \psi_{\zeta}$, for a morphism $\psi_{\zeta}: \mathcal{E}_{\mathcal{L}^{\prime}} \rightarrow \mathcal{L}^{\prime}$. The composition $\psi_{\zeta} \circ \alpha^{\prime}: \mathcal{L}^{\prime} \rightarrow \mathcal{L}^{\prime}$ is trivial, because otherwise $\psi_{\zeta}$ would define a splitting of the first exact sequence. Therefore $\psi_{\zeta} \circ \alpha^{\prime}=0$, which shows that $\psi_{\zeta}$ factorizes through a morphism $\mathcal{L}^{\prime \vee} \otimes \mathcal{K} \rightarrow \mathcal{L}^{\prime}$. This must vanish, because $X$ has no numerical anti-canonical curves. Therefore $\varphi=\zeta \operatorname{id}_{\mathcal{E}_{\mathcal{L}^{\prime}}}$, proving that $\mathcal{E}_{\mathcal{L}^{\prime}}$ is simple.

The same method applies for the statements concerning the bundles $\mathcal{A}_{\mathcal{R}}$.

3. Let $\varphi: \mathcal{E}_{L} \rightarrow \mathcal{A}_{\mathcal{R}}$ be a morphism, and consider the diagram.

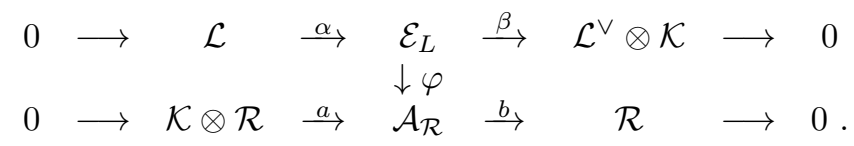

The induced morphism $b \circ \varphi \circ \alpha$ is a section of the holomorphic line bundle $\mathcal{L}^{\vee} \otimes \mathcal{R}$. Since $X$ has no numerically trivial curves, this morphism can only be trivial if $\mathcal{L}=\mathcal{R}$, and in this case, it is a multiple $\zeta \mathrm{id}_{\mathcal{R}}$ of the identity map. If $\zeta \neq 0, \varphi \circ \alpha$ will provide a splitting of the second line. Therefore $b \circ \varphi \circ \alpha=0$, hence $\varphi$ induces a morphism $\mathcal{L}^{\vee} \otimes \mathcal{K} \rightarrow \mathcal{R}$, which will vanish when $X$ admits no numerically anticanonical curves.

This shows that $\varphi$ factorizes as $\varphi=a \circ \psi$ for a morphism $\psi: \mathcal{E}_{L} \rightarrow \mathcal{K} \otimes \mathcal{R}$, so it cannot be an isomorphism.

4. An element $\varphi \in H^{0}\left(\mathcal{E} n d_{0}\left(\mathcal{E}_{\mathcal{L}}\right) \otimes \mathcal{K}\right)=H^{2}\left(\mathcal{E} n d_{0}\left(\mathcal{E}_{\mathcal{L}}\right)\right)^{\vee}$ defines a morphism $\varphi: \mathcal{E}_{\mathcal{L}} \rightarrow \mathcal{E}_{\mathcal{L}} \otimes \mathcal{K}$

Consider the diagram

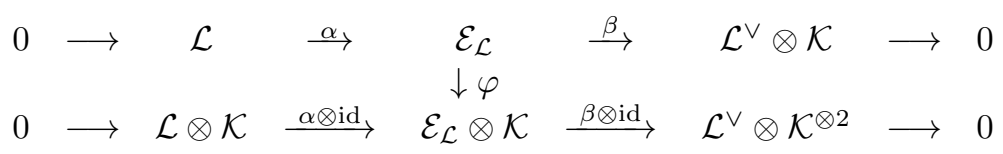

When $X$ has no bicanonical divisors, one has $(\beta \otimes$ id $) \circ \varphi \circ \alpha=0$, so $\varphi$ induces a morphism $\mathcal{L}^{\vee} \otimes \mathcal{K} \rightarrow \mathcal{L}^{\vee} \otimes \mathcal{K}^{\otimes 2}$, which must vanish, because $H^{0}(\mathcal{K})=0$. Therefore, $\varphi$ factorizes as $\varphi=(\alpha \otimes$ id $) \circ \psi$ for a morphism $\psi: \mathcal{E}_{\mathcal{L}} \rightarrow \mathcal{L} \otimes \mathcal{K}$. The composition $\psi \circ \alpha$ must vanish, because $X$ has no canonical divisors.

Therefore $\psi$ is induced by a morphism $\mathcal{L}^{\vee} \otimes \mathcal{K} \rightarrow \mathcal{L} \otimes \mathcal{K}$ which can be nontrivial only when $\mathcal{L}^{\otimes 2} \simeq \mathcal{O}$.

5. We will study the Kuranishi local model of $\mathcal{M}^{s}$ at the point $\left[\mathcal{E}_{\mathcal{R}}\right]$.

The bundle $\mathcal{E} n d_{0}\left(\mathcal{E}_{\mathcal{R}}\right)$ fits in the following diagram with exact horizontal and 
vertical lines.

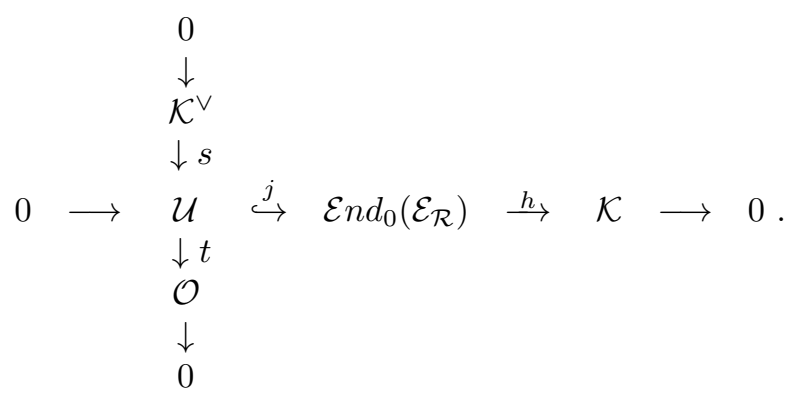

Here $\mathcal{U}$ is just the kernel of the morphism $h: \mathcal{E} n d_{0}\left(\mathcal{E}_{\mathcal{R}}\right) \rightarrow \mathcal{K}$ given by

$$
h: \varphi \mapsto \beta \circ \varphi \circ \alpha
$$

(with the notations of 1 . and 2.), i.e. the sheaf of trace free endomorphisms of $\mathcal{E}_{\mathcal{R}}$ which leave invariant the filtration $0 \subset \alpha(\mathcal{R}) \subset \mathcal{E}_{\mathcal{R}}$. The fiber $\mathcal{U}(x)$ is just the Lie algebra of the parabolic subgroup of $S L\left(\mathcal{E}_{\mathcal{R}}(x)\right)$ which is the stabilizer of the line $\alpha(\mathcal{R})(x) \subset \mathcal{E}_{\mathcal{R}}(x)$.

The morphism $s: \mathcal{K}^{\vee}=\mathcal{H o m}(\mathcal{K} \otimes \mathcal{R}, \mathcal{R}) \longrightarrow \mathcal{U}$ is given by

$$
\psi \mapsto \alpha \circ \psi \circ \beta,
$$

and $t$ is defined by

$$
\left.u\right|_{\mathcal{R}}=t(u) \operatorname{id}_{\mathcal{R}} .
$$

The vertical extension is non-trivial (it is just the extension defining $\mathcal{E}_{\mathcal{R}}$ tensorized by $\left.\mathcal{K}^{\vee} \otimes \mathcal{R}\right)$, so one gets immediately

$$
H^{1}(\mathcal{U})=H^{1}(\mathcal{O}) \simeq \mathbb{C}, H^{2}(\mathcal{U})=0 .
$$

The long exact sequence associated with the horizontal line gives an exact sequence

$$
0 \longrightarrow H^{1}(\mathcal{U})=H^{1}(\mathcal{O}) \longrightarrow H^{1}\left(\mathcal{E} n d_{0}\left(\mathcal{E}_{\mathcal{R}}\right)\right) \longrightarrow H^{1}(\mathcal{K}) \longrightarrow 0
$$

and $H^{2}\left(\mathcal{E} n d_{0}\left(\mathcal{E}_{\mathcal{R}}\right)\right) \simeq H^{2}(\mathcal{K}) \simeq \mathbb{C}$.

The germ of $\mathcal{M}^{s}$ at $\left[\mathcal{E}_{\mathcal{R}}\right]$ is isomorphic to the vanishing locus of a holomorphic map $H^{1}\left(\mathcal{E} n d_{0}\left(\mathcal{E}_{\mathcal{R}}\right)\right) \supset V \stackrel{\chi}{\longrightarrow} H^{2}\left(\mathcal{E} n d_{0}\left(\mathcal{E}_{\mathcal{R}}\right)\right)$, defined in a neighborhood of $V$ of 0 , and having the properties

$$
\chi(0)=0, d_{0} \chi=0, d_{0}^{(2)} \chi(u, v)=[u, v]
$$

where $[\cdot, \cdot]$ is the symmetric bilinear map

$$
H^{1}\left(\mathcal{E} n d_{0}\left(\mathcal{E}_{\mathcal{R}}\right)\right) \times H^{1}\left(\mathcal{E} n d_{0}\left(\mathcal{E}_{\mathcal{R}}\right)\right) \rightarrow H^{2}\left(\mathcal{E} n d_{0}\left(\mathcal{E}_{\mathcal{R}}\right)\right)
$$

induced by the commutator map on trace free endomorphisms. 
Using a fiber splitting $\mathcal{E}_{\mathcal{R}}(x)=\mathcal{R}(x) \oplus[\mathcal{K} \otimes \mathcal{R}](x)$ of the exact sequence (4), one has

$$
\left[\left(\begin{array}{cc}
\zeta & \lambda \\
k & -\zeta
\end{array}\right),\left(\begin{array}{cc}
z & l \\
0 & -z
\end{array}\right)\right]=\left(\begin{array}{cc}
* & * \\
2 k z & *
\end{array}\right), \forall z, \zeta \in \mathbb{C}, l, \lambda \in \mathcal{K}^{\vee}(x), k \in \mathcal{K}(x) .
$$

This shows that, in any point $x \in X$, it holds

$$
h([\varphi, u])=2 h(\varphi) t(u), \forall \varphi \in \mathcal{E} n d_{0}\left(\mathcal{E}_{\mathcal{R}}\right)_{x} u \in \mathcal{U}_{x} .
$$

Therefore, via the isomorphism $h_{*}: H^{2}\left(\mathcal{E}_{n d_{0}}\left(\mathcal{E}_{\mathcal{R}}\right)\right) \rightarrow H^{2}(\mathcal{K})$, one can write

$$
\left[\varphi, j_{*}(u)\right]=2 h_{*}(\varphi) \cup t_{*}(u), \forall \varphi \in H^{1}\left(\mathcal{E}_{n} d_{0}\left(\mathcal{E}_{\mathcal{R}}\right)\right), \forall u \in H^{1}(\mathcal{U}) .
$$

Via an isomorphism $H^{1}\left(\mathcal{E} n d_{0}\left(\mathcal{E}_{\mathcal{R}}\right)\right)=H^{1}(\mathcal{K}) \oplus H^{1}(\mathcal{O}) \simeq \mathbb{C}^{2}$ defined by a splitting of (6), the quadratic form associated with the second derivative $d_{0}^{(2)} \chi$ will have the form

$$
(k, \zeta) \mapsto 2 k \zeta+\alpha k^{2} .
$$

Since the first derivative vanishes, it follows that the vanishing locus $Z(\chi)$ has a simple normal crossing singularity at 0 .

It remains to prove that the map $\mathcal{L} \mapsto \mathcal{E}_{\mathcal{L}}$ parameterizes a curve in the moduli space whose tangent space at $\left[\mathcal{E}_{\mathcal{R}}\right]$ is $j_{*}\left(H^{1}(\mathcal{U})\right) \subset H^{1}\left(\mathcal{E} n d_{0}\left(\mathcal{E}_{\mathcal{R}}\right)\right)$.

Let $\Theta$ be the underlying differentiable line bundle of $\mathcal{R}$, let $\theta:=c_{1}(\Theta) \in$ $\operatorname{Tors}_{2}\left(H^{2}(X, \mathbb{Z})\right)$, and $[\mathcal{L}] \in \operatorname{Pic}^{\theta}$. Let $K$ be the underlying $\mathcal{C}^{\infty}$ differentiable bundle of $\mathcal{K}$. The bundle $\mathcal{E}_{\mathcal{L}}$ is obtained by putting on the differentiable bundle $\Theta \oplus[K \otimes \Theta]$ the holomorphic structure defined by an integrable semiconnection of the form

$$
\delta=\left(\begin{array}{cc}
\delta^{\prime} & \sigma \\
0 & \delta^{\prime \prime}
\end{array}\right) \in \mathcal{A}^{0,1}(\Theta \oplus[K \otimes \Theta]) .
$$

where $\delta^{\prime}$ defines the holomorphic structure $\mathcal{L}$ on $\Theta, \delta^{\prime \prime}$ defines the holomorphic structure $\mathcal{K} \otimes \mathcal{L}^{\vee}$ on $K \otimes \Theta \simeq K \otimes \Theta^{-1}$ and $\sigma \in A^{0,1}\left(K^{\vee}\right)$ satisfies

i) $\delta^{\prime} \circ \sigma+\sigma \circ \delta^{\prime \prime}=0$, i.e. $\sigma$ is $\bar{\partial}$-closed with respect to the holomorphic structure $\delta^{\prime} \otimes\left(\delta^{\prime \prime}\right)^{\vee}$ (which defines the holomorphic structure $\mathcal{K}^{\vee} \otimes \mathcal{L}^{\otimes 2}$ on $\left.K^{\vee}\right)$.

ii) The Dolbeault $\bar{\partial}$-cohomology class defined by $\sigma$ is non-zero.

Let $S$ be a small neighborhood of $l_{0}:=[\mathcal{R}]$ in $\operatorname{Pic}^{\theta}$ and $S \ni l \mapsto \delta_{l}^{\prime}$ a holomorphic family of integrable semiconnections ("(0,1)-connections") on $\Theta$, such that, for any $l$, the isomorphism class of the holomorphic line bundle $\mathcal{L}_{l}:=$ $\left(\Theta, \delta_{l}^{\prime}\right)$ is $l$. Put $\delta_{l}^{\prime \prime}:=\kappa \otimes\left(\delta_{l}^{\prime}\right)^{\vee}$, where $\kappa$ is the semiconnection on $K$ which defines the canonical holomorphic structure $\mathcal{K}$. The point is that, for sufficiently small $S$, we can choose $\sigma_{l}$ satisfying properties i), ii) above for $\left(\delta_{l}^{\prime}, \delta_{l}^{\prime \prime}\right)$ and depending holomorphically on $l \in V$. Indeed, since $h^{1}\left(\mathcal{K}^{\vee} \otimes \mathcal{L}_{l}^{\otimes 2}\right)$ is constant, the family of kernels of the operators $\delta_{l}^{\prime} \otimes\left(\delta^{\prime \prime}\right)_{l}^{\vee}: A^{0,1}\left(K^{\vee}\right) \rightarrow A^{0,2}\left(K^{\vee}\right)$ gives a holomorphic map from $S$ to the Grassmann manifold of closed subspaces of (a suitable Sobolev completion of) $A^{0,1}\left(K^{\vee}\right)$. 
Let $v \in T_{l_{0}}\left(\mathrm{Pic}^{\theta}\right) \backslash\{0\}$, and consider the map $\delta: S \rightarrow \mathcal{A}_{\kappa}^{0,1}(\Theta \oplus[K \otimes \Theta])$

$$
l \mapsto \delta_{l}:=\left(\begin{array}{cc}
\delta_{l}^{\prime} & \sigma_{l} \\
0 & \delta_{l}^{\prime \prime}
\end{array}\right) .
$$

The derivative $d_{l_{0}} \delta(v)$ is obviously an element in $A^{0,1}(\mathcal{U})$ which is $\bar{\partial}$-closed with respect to the holomorphic structure $\delta_{l_{0}}$ (i.e. the holomorphic structure induced from $\left.\mathcal{E} n d_{0}\left(\mathcal{E}_{\mathcal{R}}\right)\right)$, so it defines a Dolbeault cohomology class $\left[d_{l_{0}} \delta(v)\right] \in H^{1}(\mathcal{U})$. Therefore the corresponding tangent vector $w \in T_{\left[\mathcal{E}_{\mathcal{R}}\right]}\left(\mathcal{M}^{s}\right)=H^{1}\left(\mathcal{E} n d_{0}\left(\mathcal{E}_{\mathcal{R}}\right)\right)$ belongs indeed to $j_{*}\left(H^{1}(\mathcal{U})\right)$. Moreover, this vector does not vanish because, differentiating the identity $\left[\left(\Theta, \delta_{l}^{\prime}\right)\right]=l$ gives $t_{*}\left(\left[d_{l_{0}} \delta(v)\right]\right)=v$.

6. An element $\varphi \in H^{0}\left(\mathcal{E} n d_{0}\left(\mathcal{A}_{\mathcal{R}}\right) \otimes \mathcal{K}\right)=H^{2}\left(\mathcal{E} n d_{0}\left(\mathcal{A}_{\mathcal{R}}\right)\right)^{\vee}$ defines a morphism $\varphi: \mathcal{A}_{\mathcal{R}} \rightarrow \mathcal{A}_{\mathcal{R}} \otimes \mathcal{K}$.

Consider the diagram

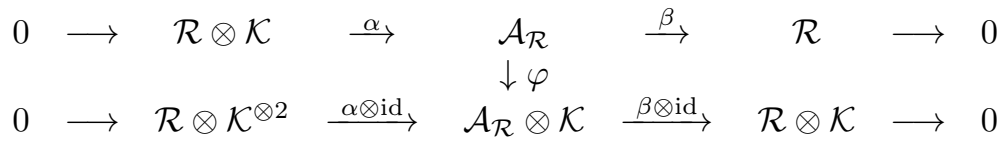

One has $(\beta \otimes \mathrm{id}) \circ \varphi \circ \alpha=0$, because, otherwise, one would get a splitting of the second exact sequence. One proceeds as in Case $b$. above, taking into account that $X$ has no canonical or bicanonical curves, and get $\varphi=0$.

7. First of all note that an extension of the type

$$
0 \longrightarrow \mathcal{M} \longrightarrow \mathcal{E} \longrightarrow \mathcal{K} \otimes \mathcal{M}^{-1} \longrightarrow 0
$$

with $c_{1}^{\mathbb{Q}}(\mathcal{M})=c_{1}^{\mathbb{Q}}(\mathcal{K})$ can be nontrivial only if the line bundle $\mathcal{M}$ has the form $\mathcal{M}=\mathcal{K} \otimes \mathcal{R}$ with $[\mathcal{R}] \in \operatorname{Tors}_{2}$ (Pic). Indeed, $\chi\left(\mathcal{K}^{\vee} \otimes \mathcal{M}^{\otimes 2}\right)=0$ by Riemann Roch, and $h^{0}\left(\mathcal{K}^{\vee} \otimes \mathcal{M}^{\otimes 2}\right)=0$ because $X$ has no numerically canonical curves. Therefore $h^{1}\left(\mathcal{K}^{\vee} \otimes \mathcal{M}^{\otimes 2}\right)$ can be non-zero only if

$$
h^{2}\left(\mathcal{K}^{\vee} \otimes \mathcal{M}^{\otimes 2}\right)=h^{0}\left(\mathcal{K}^{\otimes 2} \otimes \mathcal{M}^{\otimes-2}\right) \neq 0 .
$$

This happens if and only if $\mathcal{M}^{\otimes 2} \simeq \mathcal{K}^{\otimes 2}$, i. e. $\mathcal{M} \otimes \mathcal{K}^{-1} \in \mathrm{Tors}_{2}$ (Pic).

The surjectivity of $\mathfrak{F}$ follows now from Proposition 3.2, and the fact that trivial extensions cannot be simple; the injectivity is stated in 2 . and 3 .

Remark 3.4 The bundles $\mathcal{E}_{\mathcal{L}}, \mathcal{A}_{\mathcal{R}}$ admit a unique rank 1 subsheaf with torsion free quotient (namely $\mathcal{L}$ in the first case and $\mathcal{K} \otimes \mathcal{R}$ in the second).

Proof: Indeed, if $\mathcal{M}$ is a subsheaf with torsion free quotient of $\mathcal{E}_{\mathcal{L}}\left(\mathcal{A}_{\mathcal{R}}\right)$ it follows, as in the proof of Proposition 3.2 that $c_{1}^{\mathbb{Q}}(\mathcal{M}) \in\left\{0, c_{1}^{\mathbb{Q}}(\mathcal{K})\right\}$.

If $\mathcal{M}$ was not $\mathcal{L}$ (respectively $\mathcal{K} \otimes \mathcal{R}$ ), one would get a non-trivial morphism $\mathcal{M} \rightarrow \mathcal{K} \otimes \mathcal{L}^{-1}$ (respectively $\mathcal{M} \rightarrow \mathcal{R}$ ) which can be lifted to $\mathcal{E}_{\mathcal{L}}$ (respectively 
$\mathcal{A}_{\mathcal{R}}$ ). This would imply either the existence of curves in the forbidden rational cohomology classes, or would give a splitting of the extension which defines $\mathcal{E}_{\mathcal{L}}$ $\left(\mathcal{A}_{\mathcal{R}}\right)$.

Corollary 3.5 Let $\rho \in H^{1}\left(X, \mathbb{Z}_{2}\right) \backslash\{0\}$ and $\mathcal{L}_{\rho}$ the associated flat line bundle (see section 2.1). Let $\mathcal{E}$ be a simple bundle on $X$ with $\operatorname{det}(\mathcal{E}) \simeq \mathcal{K}, c_{2}(\mathcal{E})=0$ such that

$$
\mathcal{E} \simeq \mathcal{E} \otimes \mathcal{L}_{\rho}
$$

Then $\mathcal{E}$ is non-filtrable.

Proof: If $\mathcal{E}$ was filtrable then, by Proposition 3.3, it would be isomorphic with one of the bundles $\mathcal{E}_{\mathcal{L}}, \mathcal{A}_{\mathcal{R}}$. But, since $\mathcal{L}_{\rho}^{\otimes 2} \simeq \mathcal{O}_{X}$, one has obviously

$$
\mathcal{E}_{\mathcal{L}} \otimes \mathcal{L}_{\rho} \simeq \mathcal{E}_{\mathcal{L} \otimes \mathcal{L}_{\rho}}, \mathcal{A}_{\mathcal{R}} \otimes \mathcal{L}_{\rho} \simeq \mathcal{A}_{\mathcal{R} \otimes \mathcal{L}_{\rho}} .
$$

Since $\mathcal{L}_{\rho}$ is non-trivial (see (3) ), it follows by Proposition 3.3 that $\mathcal{E}_{\mathcal{L}} \neq \mathcal{E}_{\mathcal{L} \otimes \mathcal{L}_{\rho}}$ and $\mathcal{A}_{\mathcal{R}} \nsucceq \mathcal{A}_{\mathcal{R} \otimes \mathcal{L}_{\rho}}$. Therefore, the bundles $\mathcal{E}_{\mathcal{L}}, \mathcal{A}_{\mathcal{R}}$ do not verify (7), so they cannot by isomorphic to $\mathcal{E}$.

\subsection{Topological properties}

Proposition 3.6 The isomorphism classes $\left[\mathcal{A}_{\mathcal{R}}\right]$ and $\left[\mathcal{E}_{\mathcal{R}}\right]$ are not separable by disjoint neighborhoods in $\mathcal{M}^{s}$. More precisely, there exists an open neighborhood $C_{\mathcal{R}}^{\prime}$ of the smooth point $\left[\mathcal{A}_{R}\right]$ such that, with the notations of the previous proposition, one has

$$
C_{\mathcal{R}}^{\prime} \backslash\left\{\left[\mathcal{A}_{\mathcal{R}}\right]\right\} \subset C_{\mathcal{R}} \backslash\left\{\left[\mathcal{E}_{\mathcal{R}}\right]\right\}
$$

Proof: Let

$$
\delta_{0}=\left(\begin{array}{cc}
\delta_{0}^{\prime} & \sigma_{0} \\
0 & \delta_{0}^{\prime \prime}
\end{array}\right)
$$

be an integrable semiconnection defining the holomorphic structure $\mathcal{E}_{\mathcal{R}}$, as in the proof of Proposition 3.3, 5. Let

$$
\mathbb{C} \supset B \rightarrow \mathcal{A}_{\kappa}^{0,1}(\Theta \oplus[K \otimes \Theta]), \delta_{t}=\left(\begin{array}{cc}
\delta_{t}^{\prime} & \sigma_{t} \\
\tau_{t} & \delta_{t}^{\prime \prime}
\end{array}\right)
$$

be a holomorphic map on the open disk such that $\delta(0)=\delta_{0}, \delta_{t}$ is integrable, and $t \mapsto\left[\delta_{t}\right]$ parameterizes biholomorphically the curve $C_{\mathcal{R}}$. In particular, the Dolbeault class $v:=\left[\dot{\delta}_{0}\right] \in H^{1}\left(\mathcal{E} n d_{0}\left(\mathcal{E}_{\mathcal{R}}\right)\right)=T_{\left[\mathcal{E}_{\mathcal{R}}\right]}\left(\mathcal{M}^{s}\right)$ of the derivative $\dot{\delta}_{0}$ at 0 is a generator of the line $T_{\left[\mathcal{E}_{\mathcal{R}}\right]}\left(C_{\mathcal{R}}\right)$.

The integrability condition implies

$$
\tau_{t} \circ \delta_{t}^{\prime}+\delta_{t}^{\prime \prime} \circ \tau_{t}=0 .
$$

Differentiating at 0 and taking into account that $\tau(0)=0$, we get

$$
\dot{\tau}_{0} \circ \delta_{0}^{\prime}+\delta_{0}^{\prime \prime} \circ \dot{\tau}_{0}=0 .
$$


Since $\delta_{0}^{\prime} \otimes \delta_{0}^{\prime \prime}=\kappa$, we see that $\dot{\tau}_{0}$ is a $\kappa$-closed $K$-valued $(0,1)$-form. Its Dolbeault cohomology class is obviously $h_{*}(v)$ (with the notations in the proof of Proposition 3.3, 5.), which does not vanish, because $C_{\mathcal{R}}$ is transversal to $\Phi_{\mathcal{R}}$ in $\left[\mathcal{E}_{\mathcal{R}}\right]$.

For $t \in B \backslash\{0\}$ set

$$
g_{t}:=\left(\begin{array}{cc}
t^{\frac{1}{2}} & 0 \\
0 & \left(t^{\frac{1}{2}}\right)^{-1}
\end{array}\right) \in S L(\Theta \oplus[K \otimes \Theta]) /_{ \pm \mathrm{Id}}, \delta^{t}:=g_{t} \cdot \delta_{t}
$$

One gets easily

$$
\lim _{t \rightarrow 0} \delta^{t}=\left(\begin{array}{cc}
\delta_{0}^{\prime} & 0 \\
\dot{\tau}_{0} & \delta_{0}^{\prime \prime}
\end{array}\right)
$$

which defines precisely the holomorphic structure $\mathcal{A}_{\mathcal{R}}$, because the Dolbeault class of $\dot{\tau}_{0}$ is non-zero. It suffices to notice that $\delta_{t}$ and $\delta^{t}$ define the same point in the moduli space, for every $t \neq 0$.

Remark: An alternative proof can be obtained by studying the versal deformation of the split bundle $\mathcal{R} \oplus[\mathcal{K} \otimes \mathcal{R}]$.

Proposition 3.7 The moduli space $\mathcal{M}^{s}$ is a smooth complex manifold of dimension 1 at any point excepting the points $\left[\mathcal{E}_{\mathcal{R}}\right],[\mathcal{R}] \in \operatorname{Tors}_{2}(\mathrm{Pic})$.

Proof: If $\mathcal{M}^{s}$ was not smooth of dimension 1 at $[\mathcal{E}]$, then $H^{0}\left(\mathcal{K} \otimes \mathcal{E} n d_{0}(\mathcal{E})\right) \neq 0$. Let $\varphi \in H^{0}\left(\mathcal{K} \otimes \mathcal{E} n d_{0}(\mathcal{E})\right) \backslash\{0\}$. Regarding $\varphi$ as a $\mathcal{K}$-valued endomorphism, one obtains a section $\operatorname{det}(\varphi) \in H^{0}\left(\mathcal{K}^{\otimes 2}\right)$, which must vanish, because our surface has $\operatorname{kod}(X)=-\infty$. Therefore, $\varphi$ has rank 1 which shows that $\mathcal{E}$ is filtrable. The claim follows now from Proposition 3.3. 4., 5., 6.

\section{A moduli space of stable bundles}

\subsection{Classifying filtrable stable bundles}

Let $g$ be a Gauduchon metric on $X$, and let $\mathcal{M}^{\text {st }}:=\mathcal{M}_{g}^{\text {st }}(0, \mathcal{K})$ be the moduli space of $g$-stable bundles with $c_{2}=0$ and det $=\mathcal{K}$.

We denote by $\mathrm{Pic}_{<t}^{c}\left(\mathrm{Pic}_{<t}^{T}\right.$ ) the subset of $\mathrm{Pic}^{c}$ (respectively $\mathrm{Pic}^{T}$ ) defined by the inequality $\operatorname{deg}_{g}(\mathcal{L})<t$. Each $\operatorname{Pic}_{<t}^{c}$ is an open pointed disk, i.e. an open disk minus a point; $\mathrm{Pic}_{<t}^{T}$ is a finite union of pointed disks. We define similarly the spaces

$$
\operatorname{Pic}_{\leq t}^{c}, \operatorname{Pic}_{\leq t}^{T}, \operatorname{Pic}_{=t}^{c}, \operatorname{Pic}_{=t}^{T},\left[\operatorname{Pic}^{c}\right]_{\leq t}^{\geq s},\left[\operatorname{Pic}^{T}\right]_{\leq t}^{\geq s} .
$$

Assumption: We assume that the metric $g$ was chosen such that $\operatorname{deg}_{g}(\mathcal{K}) \neq 0$. 
The set of Gauduchon metrics satisfying this assumption is open and dense. Indeed, since $c_{1}(\mathcal{K})^{2} \neq 0$, it follows that, perturbing $\omega_{g}$ with a generic, closed, cohomologically non-trivial, small $(1,1)$-form, will yield such a metric. The same argument applies to any given holomorphic line bundle $\mathcal{L}$ with non-trivial $c_{1}(\mathcal{L})^{2}$. Note however, that, in general, it is very difficult to see whether $\operatorname{deg}_{g}(\mathcal{L})$ is always positive, always negative, or can have both signs as $g$ varies in the space of Gauduchon metrics. For instance, if the $\partial \bar{\partial}$-Chern class of $\mathcal{L}$ is a rational combination with positive coefficients of $\partial \bar{\partial}$-Chern classes of line bundles associated with curves, the degree $\operatorname{deg}_{g}(\mathcal{L})$ will be always positive.

On the other hand - as noticed by one of the two referees - using Buchdahl's ampleness criterion for non-Kählerian surfaces $\mathrm{Bu2}$, one can prove that both signes are possible when $c_{1}(\mathcal{L})^{2} \neq 0$ and $X$ has no curves. Therefore, under this hypothesis on $X$, one can choose $g$ such that $\operatorname{deg}_{g}(\mathcal{K})<0$ and continue the proof treating only this case. Since the case $\operatorname{deg}_{g}(\mathcal{K})>0$ is not much more difficult than the other one, we will not follow this way.

Set $\mathfrak{k}:=\operatorname{deg}_{g}(\mathcal{K}) / 2$. Remark 3.4 shows that the stability of $\mathcal{E}_{\mathcal{L}}$ reduces to the condition $\operatorname{deg}_{g}(\mathcal{L})<\mathfrak{k}$, and the stability of $\mathcal{A}_{\mathcal{R}}$ reduces to the condition $\operatorname{deg}_{g}(\mathcal{K})<\mathfrak{k}$. Therefore

Theorem 4.1 Under the assumptions and with the notations of Proposition 3.3 the following holds:

1. $\mathcal{E}_{\mathcal{L}}$ is g-stable if and only if $\operatorname{deg}_{g}(\mathcal{L})<\mathfrak{k}$.

2. When $\operatorname{deg}_{g} \mathcal{K}<0$, the bundles $\mathcal{A}_{\mathcal{R}},[\mathcal{R}] \in \operatorname{Tors}_{2}$ (Pic) are all stable. When $\operatorname{deg}_{g} \mathcal{K}>0$, they are not stable.

3. If $\operatorname{deg}_{g}(\mathcal{K})<0$, then the restriction

$$
\left.\mathfrak{F}\right|_{\operatorname{Pic}_{<\mathfrak{k}}^{T} \amalg \operatorname{Tors}_{2}(\mathrm{Pic})}: \operatorname{Pic}_{<\mathfrak{k}}^{T} \coprod \operatorname{Tors}_{2}(\mathrm{Pic}) \longrightarrow \mathcal{M}^{s}
$$

maps bijectively $\mathrm{Pic}_{<\mathfrak{k}}^{T} \amalg \operatorname{Tors}_{2}(\mathrm{Pic})$ on the filtrable part of $\mathcal{M}^{\text {st }}$. The image of the subspace $\mathrm{Pic}_{<\mathfrak{k}}^{T}$ is open in $\mathcal{M}^{\text {st }}$.

4. If $\operatorname{deg}_{g}(\mathcal{K})>0$, then the restriction

$$
\left.\mathfrak{F}\right|_{\operatorname{Pic}_{<\mathfrak{k}}^{T}}: \operatorname{Pic}_{<\mathfrak{k}}^{T} \longrightarrow \mathcal{M}^{s}
$$

maps bijectively $\operatorname{Pic}_{<\mathfrak{k}}^{T}$ on the filtrable part of $\mathcal{M}^{\text {st }}$. The image of the subspace $\mathrm{Pic}_{<\mathfrak{k}}^{T} \backslash \mathrm{Tors}_{2}(\mathrm{Pic})$ is open in $\mathcal{M}^{\text {st }}$.

Throughout the rest of the paper $X$ will always denote a class VII surface with $b_{2}=1$ satisfying the hypothesis of Proposition 3.3 


\subsection{A collar around the reductions}

Let $E$ be a Hermitian 2-bundle with $c_{2}(E)=0, \operatorname{det}(E)=K$, and let $a$ be the Chern connection of the pair $(\mathcal{K}, \operatorname{det}(h))$.

For every $c \in \operatorname{Tors}\left(H^{2}(X, \mathbb{Z})\right.$ ) one has a unique (up to the gauge group $S U(E)$ ) orthogonal decomposition

$$
E=L \oplus\left[K \otimes L^{\vee}\right],
$$

where $L$ is a Hermitian line bundle of Chern class $c$, and these are the only splittings of $E$ (by the same computation as in the proof of Proposition 3.2). Every reducible connection $A \in \mathcal{M}_{a}^{\mathrm{ASD}}(E)$ is equivalent to a direct sum $b \oplus(a \otimes$ $b^{\vee}$ ), where $b$ is a Hermitian connection on $L$ such that

$$
\left(2 F_{b}-F_{a}\right)^{+}=0
$$

The condition $\left(2 F_{b}-F_{a}\right)^{+}=0$ means that $2 F_{b}-F_{a}$ is an ASD form so, being closed, it coincides with the unique harmonic representative $r$ of the de Rham cohomology class $-2 \pi i c_{1}^{\mathrm{DR}}(X)$. The equation (8) becomes

$$
F_{b}=\frac{1}{2}\left(F_{a}+r\right) \text {. }
$$

Let $\mathcal{N}_{c}$ be the moduli space of solutions $b$ of (9) modulo the gauge group $\mathcal{C}^{\infty}\left(X, S^{1}\right)$ of $L$. It is well-known that the moduli space of Hermitian connections of fixed curvature on a Hermitian line bundle over an arbitrary compact manifold $V$ is a $\mathbb{T}_{V}$-torsor 1 , where $\mathbb{T}_{V}$ is the torus

$$
\mathbb{T}_{V}:=i H^{1}(V, \mathbb{R}) / 2 \pi i H^{1}(V, \mathbb{Z}) .
$$

Therefore it is (non-canonically) isomorphic to this torus. In our case, $\mathbb{T}_{X}$ is obviously a circle. Therefore

Proposition 4.2 The subspace $\mathcal{M}^{\mathrm{red}} \subset \mathcal{M}_{a}^{\mathrm{ASD}}(E)$ of reducible solutions decomposes as a disjoint union

$$
\mathcal{M}^{\text {red }}=\coprod_{c \in \operatorname{Tors}\left(H^{2}(X, \mathbb{Z})\right)} \mathcal{M}_{c}^{\text {red }}
$$

where $\mathcal{M}_{c}^{\text {red }}$ is the moduli space of solutions which admit a Hermitian line bundle $L$ with $c_{1}(L)=c$ as parallel summand. Each $\mathcal{M}_{c}^{\text {red }}$ is naturally isomorphic to the circle $\mathcal{N}_{c}$.

Our next purpose is to understand the topology of $\mathcal{M}_{a}^{\mathrm{ASD}}(E)$ around the reducible locus $\mathcal{M}^{\text {red }}$. It is very natural (and almost obvious) that the circle $\mathcal{N}_{c}$ is contained in the closure of the pointed disk $\mathfrak{F}\left(\mathrm{Pic}_{<\mathfrak{k}}^{c}\right)$, so that the union

\footnotetext{
${ }^{1}$ In general, a $G$-torsor is a set $\Gamma$ endowed with a free transitive $G$-action. Fixing a point $\gamma \in \Gamma$ gives an identification $\Gamma \simeq G$, and any two such identifications differ by a $G$-translation.
} 
$\mathfrak{F}\left(\operatorname{Pic}_{<\mathfrak{k}}^{c}\right) \cup \mathcal{N}_{c}$ becomes a closed pointed disk. Indeed, the limit of the stable bundle $\mathcal{E}_{\mathcal{L}}$ as $\mathcal{L} \rightarrow \mathcal{L}_{0}$ (with $\operatorname{deg}_{g}(\mathcal{L})_{0}=\mathfrak{k}$ ) should be the polystable bundle $\mathcal{L}_{0} \oplus\left[\mathcal{K} \otimes \mathcal{L}_{0}^{\vee}\right]$, and the circle of polystable bundles of this form can be naturally identified with $\mathcal{N}_{c}$.

However, the local structure of a moduli space of polystable bundles around the non-stable points can be in general very complicated, and it is not a subject available in the literature for our non-Kählerian framework. Hence we will indicate a simple ad-hoc argument.

Lemma 4.3 Let $A$ be a reducible solution in $\mathcal{A}_{a}^{\mathrm{ASD}}(E)$. Then the harmonic space $\mathbb{H}_{A}^{2}$ of the deformation elliptic complex associated with $A$ vanishes.

Proof: Let $L \oplus\left[K \otimes L^{\vee}\right]$ be the decomposition of $E$ in $A$-parallel factors, and let $A=b \oplus c$ be the corresponding splitting of $A$. One has an $A$-parallel splitting

$$
\operatorname{su}(E)=(X \times[i \mathbb{R}]) \oplus\left[K^{\vee} \otimes L^{2}\right]
$$

and the induced connections on the summands are the trivial connection and $f:=b \otimes c^{\vee}=a^{\vee} \otimes b^{\otimes 2}$ respectively. The connections $b$ and $f$ are integrable, so they define holomorphic structures $\mathcal{L}, \mathcal{F}$ on $L$ and $F:=K^{\vee} \otimes L^{2}$. Note $f$ is an ASD connection (or, equivalently, a Hermitian-Einstein connection of vanishing Einstein constant), and that

$$
\operatorname{deg}_{g}(\mathcal{L})=\mathfrak{k}, \operatorname{deg}_{g}(\mathcal{F})=0 .
$$

As in the Kählerian case $\left[\mathrm{K}\right.$, the idea is to compare the $d^{+}$-complex of $f$ with the Dolbeault complex of $\bar{\partial}_{f}$. We have the diagram

$$
\begin{aligned}
& 0 \rightarrow 0 \quad \longrightarrow \quad A^{10}(F) \stackrel{\left(\partial_{f}, p^{\omega} \bar{\partial}_{f}\right)}{\longrightarrow} A^{20}(F) \oplus A^{0}(F) \omega_{g} \quad \rightarrow \quad 0 \\
& \downarrow \quad \downarrow i \quad \downarrow j \\
& 0 \rightarrow A^{0}(F) \stackrel{d_{f}}{\longrightarrow} A^{1}(F) \quad \stackrel{d_{f}^{+}}{\longrightarrow} \quad A_{+}^{2}(F) \quad \rightarrow 0 \\
& \| \quad \downarrow p^{01} \quad \downarrow p^{02} \\
& 0 \rightarrow A^{0}(F) \stackrel{\bar{\partial}_{f}}{\longrightarrow} A^{01}(F) \quad \stackrel{\bar{\partial}_{f}}{\longrightarrow} \quad A^{02}(F) \quad \rightarrow \quad 0
\end{aligned}
$$

where $i, j$ are the obvious inclusions, and $p^{01}, p^{02}, p^{\omega}$ the obvious projections.

The cohomology of the third line is just the cohomology of the holomorphic bundle $\mathcal{F}$ associated with the semiconnection $\bar{\partial}_{f}$. But

$$
H^{2}(\mathcal{F})=H^{2}\left(\mathcal{K}^{\vee} \otimes \mathcal{L}^{\otimes 2}\right)=H^{0}\left(\mathcal{K}^{\otimes 2} \otimes \mathcal{L}^{\otimes-2}\right)^{\vee}=0
$$

because our surface does not have numerically bicanonical curves. It suffices to show that the second cohomology of the first line vanishes.

Let $\left(a^{20}, \varphi \omega_{g}\right) \in \operatorname{coker}\left(\partial_{f}, p^{\omega} \bar{\partial}_{f}\right)$. This implies

$$
\partial_{f}^{*}\left(a^{20}\right)+\bar{\partial}_{f}^{*}\left(\varphi \omega_{g}\right)=0
$$


hence, since $a^{20}$ and $\omega_{g}$ are self-dual with respect to the ( $\mathbb{C}$-linear) Hodge operator,

$$
\bar{\partial}_{f}\left(a^{20}\right)+\partial_{f}\left(\varphi \omega_{g}\right)=0 .
$$

We get $2 \bar{\partial}_{f} \partial_{f}\left(\varphi \omega_{g}\right)=0$. We claim that the kernel of the second order elliptic operator $q_{f}: A^{0}(F) \rightarrow A^{0}(F)$ defined by $q_{f}(\psi):=i * \bar{\partial}_{f} \partial_{f}\left(\psi \omega_{g}\right)$ vanishes. The adjoint of this operator is $p_{f}=i \Lambda_{g} \bar{\partial}_{f} \partial_{f}$ (see [Bu1], [LT1] p. 225). Taking into account $i \Lambda_{g}\left(\bar{\partial}_{f} \partial_{f}+\partial_{f} \bar{\partial}_{f}\right)=0$ (because $f$ is ASD), one gets the identity

$$
i \Lambda_{g} \bar{\partial} \partial|\psi|^{2}=\left(p_{f}(\psi), \psi\right)-\left|d_{f}(\psi)\right|^{2}+\left(\psi, p_{f}(\psi)\right)
$$

(compare with the computation in [LT1, Lemma 1.2.5, p. 31). By the maximum principle, it follows that $\operatorname{ker}\left(p_{f}\right)=\operatorname{ker}\left(d_{f}\right)$, which vanishes, because $\mathcal{F}$ is nontrivial. Since both operators have index 0 , we also get $\operatorname{ker}\left(q_{f}\right)=0$. Therefore $\varphi=0$, which implies that $\bar{\partial}_{f}\left(a^{20}\right)=0$, so $a^{20}$ defines a holomorphic section in $\mathcal{K} \otimes \mathcal{F}=\mathcal{L}^{\otimes 2}$. It follows that $a^{20}=0$, because the holomorphic line bundle $\mathcal{L}^{\otimes 2}$ is non-trivial (has non-vanishing degree) and our surface has no numerically trivial curve.

For every holomorphic line bundle $\mathcal{L}$ we denote by $b_{\mathcal{L}}$ its unique HermitianEinstein connection.

Proposition 4.4 The extension $\overline{\mathfrak{F}}: \operatorname{Pic}_{\leq \mathfrak{k}}^{T} \rightarrow \mathcal{M}_{a}^{\mathrm{ASD}}(E)$ of $\left.\mathfrak{F}\right|_{\mathrm{Pic}_{<\mathfrak{k}}^{T}}$ defined by

$$
\overline{\mathfrak{F}}([\mathcal{L}]):=\left[b_{\mathcal{L}} \oplus\left(a \otimes b_{\mathcal{L}}^{\vee}\right)\right], \quad \forall[\mathcal{L}] \in \operatorname{Pic}_{=\mathfrak{k}}^{T}
$$

maps homeomorphically $\operatorname{Pic}_{\leq \mathfrak{k}}^{T} \backslash \operatorname{Tors}_{2}(\mathrm{Pic})$ on an open subspace of $\mathcal{M}_{a}^{\mathrm{ASD}}(E)$. In particular $\mathcal{M}_{a}^{\mathrm{ASD}}(E)$ has the structure of a real 2-manifold with boundary near $\mathcal{M}^{\text {red }}$.

Proof: By the standard Kobayashi-Hitchin correspondence between stable bundles and Hermitian-Einstein connections [LT1] we get immediately that the restriction $\left.\mathfrak{F}\right|_{\operatorname{Pic}_{<\mathfrak{e}}^{T} \backslash \operatorname{Tors}_{2}(\mathrm{Pic})}$ is an open embedding.

It remains to prove that $\overline{\mathfrak{F}}$ is a local homeomorphism near the union of circles $\operatorname{Pic}_{=\mathfrak{k}}^{T}$.

Let $\varepsilon>0$. For an integrable connection $A$ denote by $\mathcal{E}_{A}$ the holomorphic bundle defined by the semiconnection $\bar{\partial}_{A}$. We define

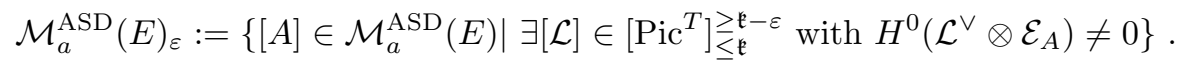

By elliptic semicontinuity it follows easily that $\mathcal{M}_{a}^{\mathrm{ASD}}(E)_{\varepsilon}$ is a closed (hence compact by Corollary (1.5) subspace of $\mathcal{M}_{a}^{\mathrm{ASD}}(E)$.

The map

$$
\lambda_{\varepsilon}: \mathcal{M}_{a}^{\mathrm{ASD}}(E)_{\varepsilon} \longrightarrow\left[\mathrm{Pic}^{T}\right]_{\leq \mathfrak{k}}^{\geq \mathfrak{k}-\varepsilon}
$$

\footnotetext{
${ }^{2} \mathrm{I}$ am grateful to one of the two referees for pointing me out an error (caused by a missing term) in this part of the proof, and for indicating a correct argument.
} 
defined by

$$
[A] \mapsto \text { the unique }[\mathcal{L}] \in \operatorname{Pic}^{T} \text { such that } H^{0}\left(\mathcal{L}^{\vee} \otimes \mathcal{E}_{A}\right) \neq 0
$$

is continuous, by elliptic semicontinuity again, and bijective. The uniqueness of $\mathcal{L}$ follows easily from our hypothesis (non-existence of numerically trivial curves). Therefore, by an elementary topological lemma, this map is a homeomorphism. By definition, this homeomorphism is the inverse of the restriction $\left.\overline{\mathfrak{F}}\right|_{\left[\mathrm{Pic}^{T}\right]_{\leq \mathfrak{e}}^{\geq \mathfrak{e}-\varepsilon}}$. It suffices to prove that

Claim: $\mathcal{M}_{a}^{\mathrm{ASD}}(E)_{\varepsilon}$ is a neighborhood of the reducible part $\mathcal{M}^{\text {red }}$.

Let $A:=b \oplus c$ be a reducible connection in $\mathcal{A}_{a}^{\mathrm{ASD}}(E)$ as in the proof of Lemma 4.3, $E=L \oplus\left[K \otimes L^{\vee}\right]$ be the corresponding $A$-parallel decomposition, $F:=K^{\vee} \otimes L^{\otimes 2}, f:=a^{\vee} \otimes b^{\otimes 2}$, and $\mathcal{F}$ the corresponding holomorphic line bundle.

Consider the space $\mathcal{S}$ of solutions of the following elliptic differential system

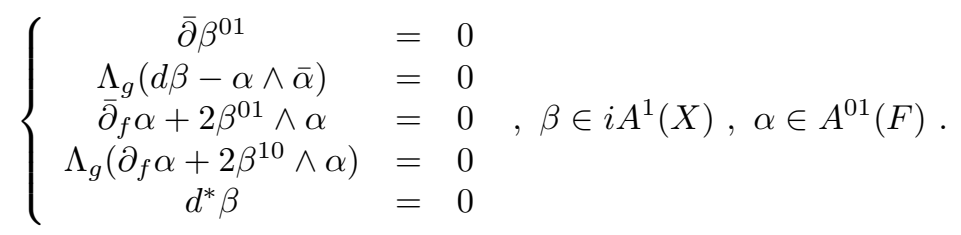

This space comes with a natural $S^{1}$-action:

$$
(\zeta,(\beta, \alpha)) \mapsto\left(\beta, \zeta^{2} \alpha\right)
$$

The linearization of (10) at 0 is

$$
\left\{\begin{array}{l}
d^{+} \beta=0 \\
\bar{\partial}_{f} \alpha=0, \quad d^{*} \beta=0 \\
\Lambda_{g} \partial_{f} \alpha=0
\end{array},\right.
$$

so the tangent space $T_{0}(\mathcal{S})$ can be identified with $i H^{1}(X, \mathbb{R}) \oplus H^{1}(\mathcal{F})$. Indeed, by the maximum principle it is easy to prove that any $(0,1)$-Dolbeault cohomology class of $\mathcal{F}$ has a unique representative $\alpha$ with $\Lambda_{g} \partial_{f} \alpha=0$ (see the proof of Lemma 4.3). The map defined by the left hand side of the system (10) is a submersion at 0 , hence $\mathcal{S}$ is a smooth manifold of dimension 3 around 0 .

We define the map $\mathfrak{A}: \mathcal{S} \rightarrow \mathcal{A}_{a}^{\mathrm{ASD}}(E)$ by

$$
\mathfrak{A}(\beta, \alpha):=\left(\begin{array}{cc}
b+\beta & \alpha \\
-\bar{\alpha} & c-\beta
\end{array}\right) .
$$

We consider $\mathcal{A}_{a}^{\mathrm{ASD}}(E)$ as an $S^{1}$-space via $\zeta \mapsto\left(\begin{array}{cc}\zeta & 0 \\ 0 & \zeta^{-1}\end{array}\right) \in \mathrm{SU}(E)$. Note that, by Lemma 4.3 , $\mathcal{A}_{a}^{\mathrm{ASD}}(E)$ is a smooth Banach manifold at $A$ (after suitable Sobolev completion). It is easy to check that 
1. $\mathfrak{A}$ is $S^{1}$ equivariant and $\mathfrak{A}(0)=A$,

2. $\mathfrak{A}$ is an immersion at 0 ,

3. $\mathfrak{A}_{*}\left(T_{0}(\mathcal{S})\right)$ is a complement of $T_{A}[A]$ in $T_{A}\left(\mathcal{A}_{a}^{\mathrm{ASD}}(E)\right)$; in particular $\operatorname{im}(\mathfrak{A})$ is transversal in $A$ at the $\mathrm{SU}(E)$-orbit $[A]=\mathrm{SU}(E) \cdot A$.

It follows that $\operatorname{im}(\mathfrak{A})$ is mapped on a neighborhood of $[A]$ in $\mathcal{M}_{a}^{\mathrm{ASD}}(E)$ and that $\mathcal{S} / S^{1}$ is a local model for the moduli space $\mathcal{M}_{a}^{\mathrm{ASD}}(E)$ at $[A]$. Therefore, in order to prove our claim, it suffices to show that, when $(\beta, \alpha) \in \mathcal{S}$ are sufficiently small, then $[\mathfrak{A}(\beta, \alpha)]$ belongs to $\mathcal{M}_{a}^{\mathrm{ASD}}(E)_{\varepsilon}$.

But, by construction, since $\bar{\alpha}$ is of type $(1,0)$, the summand $L$ of $E$ is always a holomorphic sub line bundle of the holomorphic bundle $\mathcal{E}_{\mathfrak{A}(\beta, \alpha)}$. The induced holomorphic structure on this summand is defined by the semiconnection $\bar{\partial}_{b}+$ $\beta^{01}$. The second equation in the system (10) shows that the degree of this holomorphic structure is just $\|\alpha\|_{L^{2}}^{2}$. Therefore, this degree becomes smaller than $\varepsilon$ for $\alpha$ sufficiently small.

\subsection{The missing centers}

Denote by $\mathrm{Pic}_{-}^{c}$, respectively $\mathrm{Pic}_{+}^{c}$, the space (isomorphic to $\mathbb{C}$ ) obtained by adding formally a point $0_{c}$ (respectively $\infty_{c}$ ) to $\mathrm{Pic}^{c}$ such that the limits

$$
\lim _{\substack{c_{1}(\mathcal{L})=c \\ \operatorname{deg}_{g}(\mathcal{L}) \rightarrow-\infty}}[\mathcal{L}]=0_{c}, \quad \lim _{\substack{c_{1}(\mathcal{L})=c \\ \operatorname{deg}_{g}(\mathcal{L}) \rightarrow+\infty}}[\mathcal{L}]=\infty_{c}
$$

exist. Set

$$
\operatorname{Pic}_{ \pm}^{T}:=\bigcup_{c \in \operatorname{Tors}\left(H^{2}(X, \mathbb{Z})\right)} \operatorname{Pic}_{ \pm}^{c} .
$$

As in the previous section, we introduce the subspaces

$$
\left[\mathrm{Pic}_{-}^{c}\right]_{<t},\left[\mathrm{Pic}_{-}^{T}\right]_{<t},\left[\mathrm{Pic}_{-}^{c}\right]_{\leq t},\left[\mathrm{Pic}_{-}^{T}\right]_{\leq t},\left[\mathrm{Pic}_{+}^{c}\right]_{>t},\left[\mathrm{Pic}_{+}^{T}\right]_{>t},\left[\mathrm{Pic}_{+}^{c}\right]_{\geq t},\left[\mathrm{Pic}_{+}^{T}\right]_{\geq t} .
$$

Proposition 4.5 $1 . \mathcal{M}^{\text {st }}$ is a complex space of pure dimension 1, which is smooth in the case $\operatorname{deg}_{g}(\mathcal{K})<0$ and whose singular locus is the finite set $\left\{\left[\mathcal{E}_{\mathcal{R}}\right]\right\}_{[R] \in \text { Tors }_{2} \text { (Pic) }}$ in the case $\operatorname{deg}_{g}(\mathcal{K})>0$.

2. The map

$$
\overline{\mathfrak{F}}: \operatorname{Pic}_{\leq \mathfrak{k}}^{T} \longrightarrow \mathcal{M}_{a}^{\mathrm{ASD}}(E)=\overline{\mathcal{M}^{\mathrm{st}}}
$$

extends to a map $\tilde{\mathfrak{F}}:\left[\mathrm{Pic}_{-}^{T}\right]_{\leq \mathfrak{k}} \longrightarrow \mathcal{M}_{a}^{\mathrm{ASD}}(E)=\overline{\mathcal{M}^{\mathrm{st}}}$ which is holomorphic on $\left[\mathrm{Pic}_{-}^{T}\right]_{<\mathfrak{k}}$.

3. The points $\tilde{\mathfrak{F}}\left(0_{c}\right)$ are fixed under the action of $H^{1}(X, \mathbb{Z}) / 2 H^{1}(X, \mathbb{Z}) \simeq \mathbb{Z}_{2}$ on the moduli space (see sections 1.2, 2.11). 
Proof: 1 . The first statement follows directly from Proposition 3.7 and Proposition 3.3 .

2. By Corollary 1.5, the space $\mathcal{M}^{\mathrm{ASD}}$ obtained by adding to $\mathcal{M}^{\text {st }}$ the circles of polystable bundles is compact. The idea of the proof is based on the following simple fact: the only way to compactify a complex line $\mathbb{P}^{1}(\mathbb{C}) \backslash\{0\}$ as a 1 dimensional complex space is by adding a point (which a priori could be of course singular).

Consider the compact 1-dimensional complex space

$$
\mathcal{C}:=\left[\mathrm{Pic}_{+}^{T}\right]_{>\mathfrak{k}-\varepsilon} \bigcup_{\mathfrak{F}} \mathcal{M}^{\mathrm{st}}
$$

obtained by filling in disks in the direction $\operatorname{deg}_{g}(\mathcal{L}) \rightarrow \infty$. By Proposition 4.4 and the first statement in this proposition, $\mathcal{C}$ is a complex space of dimension 1 with at most $\left|\operatorname{Tors}_{2}(\mathrm{Pic})\right|$ simple (normal crossing) singularities.

The irreducible component of $\mathcal{C}$ which contains the line

$$
\left[\mathrm{Pic}_{+}^{c}\right]_{>\mathfrak{k}-\varepsilon} \bigcup_{\mathfrak{F}} \overline{\mathfrak{F}}\left(\mathrm{Pic}_{<\mathfrak{k}}^{c}\right)
$$

is an irreducible algebraic curve, so it can be embedded in a projective space $\mathbb{P}^{n}(\mathbb{C})$ such that the intersection with the hyperplane at infinity is $\left\{\infty_{c}\right\}$. Then $\left.\mathfrak{F}\right|_{\text {Pic }_{<\mathfrak{t}}^{c}}$ factorizes through a bounded $\mathbb{C}^{n}$-valued map, which can be obviously extended holomorphically in $0_{c}$.

3. Let $\otimes \rho: \mathcal{M}^{\text {st }} \rightarrow \mathcal{M}^{\text {st }}$ be the involution induced by the generator $\rho$ of $H^{1}(X, \mathbb{Z}) / 2 H^{1}(X, \mathbb{Z})$, and $\left[\mathcal{L}_{\rho}\right] \in \mathrm{Pic}^{0}$ be the line bundle associated with the representation $\pi_{1}(X) \rightarrow\{ \pm 1\} \subset S^{1} \subset \mathbb{C}^{*}$ defined by $\rho$ (see section 2.1). One has obviously the identity

$$
\otimes \rho\left(\mathfrak{F}([\mathcal{L}])=\left[\mathcal{E}_{\mathcal{L}} \otimes \mathcal{L}_{\rho}\right]=\mathfrak{F}\left(\left[\mathcal{L} \otimes \mathcal{L}_{\rho}\right]\right) .\right.
$$

It suffices now to note that

$$
\lim _{\substack{c_{1}(\mathcal{L})=c \\ \operatorname{deg}_{g}(\mathcal{L}) \rightarrow-\infty}}\left[\mathcal{L} \otimes \mathcal{L}_{\rho}\right]=0_{c},
$$

because $\operatorname{deg}_{g}\left(\mathcal{L}_{\rho}\right)=0$.

The fact that the points $\tilde{\mathfrak{F}}\left(0_{c}\right)$ are fixed under the involution $\otimes \rho$ is very important. Using Corollary 3.5] and Proposition 3.7, one obtains

Corollary 4.6 The point $\tilde{\mathfrak{F}}\left(0_{c}\right)$ corresponds to a non-filtrable bundle, in particular it is a smooth point in the moduli space $\mathcal{M}^{\text {st }}$.

Let $\mathcal{E}_{c}$ be a bundle in the isomorphy class $\tilde{\mathfrak{F}}\left(0_{c}\right)$. The isomorphism $\mathcal{E}_{c} \simeq \mathcal{E}_{c} \otimes \mathcal{L}_{\rho}$ shows that $\mathcal{E}_{c}$ can be obtained as push-forward of a line bundle on a bicovering. More precisely, let $\pi_{\rho}: \tilde{X}_{\rho} \rightarrow X$ be the bicovering of $X$ associated with $\rho$ (regarded as a representation $\left.\pi_{1}\left(X, x_{0}\right) \rightarrow \mathbb{Z}_{2}\right)$. 
Remark 4.7 For every $c \in \operatorname{Tors}\left(H^{2}(X, \mathbb{Z})\right)$ there exists a holomorphic line bundle $\mathcal{M}_{c}$ on $\tilde{X}_{\rho}$ such that $\mathcal{E}_{c} \simeq\left[\pi_{\rho}\right]_{*}\left(\mathcal{M}_{c}\right)$.

Remark: Our results so far show that the moduli space $\overline{\mathcal{M}^{\text {st }}}$ contains as an open set the disjoint union $\left[\coprod_{i=1}^{p-q} D_{i}\right] \amalg\left[\coprod_{j=1}^{q} P_{j}\right]$ described in section 1.3 The point $\tilde{\mathfrak{F}}\left(0_{c}\right)$ corresponding to a class $c \in \operatorname{Tors}_{2}\left(H^{2}(X, \mathbb{Z})\right)$ is the center $O$ of the corresponding "horizontal" disk $\tilde{\mathfrak{F}}\left(\left[\mathrm{Pic}_{-}^{c}\right]_{\leq \mathfrak{k}}\right)$ (denoted by $E$ in the picture). Corollary 4.6 plays an important role: it shows in particular that the center of a "vertical" disk $C_{i}^{\prime}$ (or $C_{i}$ ) cannot coincide with a center $\tilde{\mathfrak{F}}\left(0_{c}\right)$, hence such a vertical disk cannot be contained in the "horizontal" locus $\operatorname{im}(\widetilde{\mathfrak{F}})$.

\subsection{A smooth compact complex curve in the moduli space}

We can prove now:

Theorem 4.8 Let $X$ be a class VII surface with $b_{2}=1$ which does not admit any divisor $C>0$ with $c_{1}^{\mathbb{Q}}(\mathcal{O}(C)) \in\left\{0, \pm c_{1}^{\mathbb{Q}}(\mathcal{K}), 2 c_{1}^{\mathbb{Q}}(\mathcal{K})\right\}$. Let $g$ be a Gauduchon metric on $X$ such that $\operatorname{deg}_{g}(\mathcal{K}) \neq 0$.

1. Suppose $\operatorname{deg}_{g}(\mathcal{K})<0$. Then $\overline{\mathcal{M}^{\text {st }}} \backslash \tilde{\mathfrak{F}}\left(\left[\mathrm{Pic}_{-}^{T}\right]_{\leq \mathfrak{k}}\right)$ is a $\underline{\text { non-empty }}$, possibly non-connected, smooth, closed complex curve whose only filtrable points are the extensions $\left[\mathcal{A}_{R}\right],[\mathcal{R}] \in \operatorname{Tors}_{2}(\mathrm{Pic})$.

2. Suppose $\operatorname{deg}_{g}(\mathcal{K})>0$. The closure of $\overline{\mathcal{M}^{\mathrm{st}}} \backslash \tilde{\mathfrak{F}}\left(\left[\mathrm{Pic}_{-}^{T}\right]_{\leq \mathfrak{k}}\right)$ is a non-empty, possibly non-connected, smooth, closed complex curve whose only filtrable points are the extensions $\left[\mathcal{E}_{R}\right],[\mathcal{R}] \in \operatorname{Tors}_{2}(\mathrm{Pic})$.

Proof: 1. In this case, by Theorem 4.1 and Proposition 4.4 the subspace $\tilde{\mathfrak{F}}\left(\left[\mathrm{Pic}_{-}^{T}\right]_{\leq \mathfrak{k}}\right)$ is open and closed in $\overline{\mathcal{M}^{\text {st }}}$. It suffices to note that $\left[\mathcal{A}_{\mathcal{R}}\right]$ does not belong to this subspace. This follows from the injectivity of the map $\mathfrak{F}$ and Corollary 4.6 which assures that the centers $\tilde{\mathfrak{F}}\left(0_{c}\right)$ are non-filtrable.

2. For any $[\mathcal{R}] \in \operatorname{Tors}_{2}(\mathrm{Pic})$ the branch $C_{\mathcal{R}}$ passing through $\left[\mathcal{E}_{\mathcal{R}}\right]$ (see Proposition 3.3) consists of stable bundles, because stability is an open property [LT1. It suffices to show that the irreducible component containing this branch is not contained in $\tilde{\mathfrak{F}}\left(\left[\mathrm{Pic}_{-}^{T}\right]_{\leq \mathfrak{k}}\right)$. The normal crossing $C_{\mathcal{R}} \cup \Phi_{\mathcal{R}}$ is mapped injectively in the moduli space. If $C_{\mathcal{R}}$ was contained in $\tilde{\mathfrak{F}}\left(\left[\mathrm{Pic}_{-}^{T}\right]_{\leq \mathfrak{k}}\right)$, the center $\left[\mathcal{E}_{\mathcal{R}}\right]$ of this branch would coincide with an element of $\tilde{\mathfrak{F}}\left(\left[\mathrm{Pic}_{-}^{T}\right]_{\leq \mathfrak{k}}\right) \backslash \Phi_{\mathcal{R}}$, which is impossible (use again the injectivity of the map $\mathfrak{F}$ and Corollary 4.6).

Theorem 4.8 yields a closed curve $Y$ and a holomorphic morphism $Y \rightarrow \mathcal{M}^{\text {st }}$ taking both filtrable and non-filtrable values. In the next section we will see that such a morphism cannot exist. 


\section{$5 \quad$ Families of bundles parameterized by a curve}

We begin with the following result concerning the correspondence between holomorphic morphisms from a curve into a moduli space of simple bundles and holomorphic families.

Lemma 5.1 Let $X$ be a complex manifold, $E$ a differentiable rank 2 bundle on $X$, and $\mathcal{L}$ a fixed holomorphic structure on $\operatorname{det}(E)$. Let $\mathcal{M}^{\mathrm{s}}(E, \mathcal{L})$ be the moduli space of simple holomorphic structures on $E$ which induce $\mathcal{L}$ on $\operatorname{det}(E)$, modulo the complex gauge group $\mathrm{SL}(E)$.

Let $Y$ be a compact complex curve and $\mathfrak{f}: Y \rightarrow \mathcal{M}^{\mathrm{s}}(E, \mathcal{L})$ be a holomorphic morphism. There exists a line bundle $\mathcal{N}$ on $Y$ and a holomorphic 2-bundle $\mathcal{E}$ on $Y \times X$ such that

1. The family $\mathcal{E}$ induces $\mathfrak{f}$, i.e.

$$
\left[\left.\mathcal{E}\right|_{\{y\} \times X}\right]=\mathfrak{f}(y), \forall y \in Y .
$$

2. $\operatorname{det}(\mathcal{E}) \simeq p_{Y}^{*}(\mathcal{N}) \otimes p_{X}^{*}(\mathcal{L})$, where $p_{X}, p_{Y}$ are the respective projections.

Proof: The deformation theory for holomorphic bundles extends easily to holomorphic structures with fixed determinant. In particular, the germ of the moduli space $\mathcal{M}^{\mathrm{s}}(E, \mathcal{L})$ at a point $[\mathcal{E}]$ is the basis of a universal deformation of $\mathcal{E}$ in the space of holomorphic structures compatible with $\mathcal{L}$ (see Miy for the case of plain bundles). This provides an open cover $\mathcal{U}=\left(U_{i}\right)_{i \in I}$ of $Y$ and bundles $\mathcal{E}_{i}$ on $U_{i} \times X$ with isomorphisms $j_{i}: \operatorname{det}\left(\mathcal{E}_{i}\right) \simeq\left[p_{X}^{i}\right]^{*}(\mathcal{L})$, where $p_{X}^{i}: U_{i} \times X \rightarrow X$ are the projections on the $X$-factor.

If the cover $\mathcal{U}$ is sufficiently fine, on the intersections $U_{i} \cap U_{j}$ we get $S L$ isomorphisms $f_{j i}: \mathcal{E}_{i} \rightarrow \mathcal{E}_{j}$. These isomorphisms are obtained in the usual way, by noting that the sheaves $\left(p_{U_{i} \cap U_{j}}\right)_{*}\left(\mathcal{E}_{i}^{\vee} \otimes \mathcal{E}_{j}\right)$ are line bundles, which will be trivial if the cover is sufficiently fine. If the intersections $U_{i} \cap U_{j}$ are simply connected one can find sections in these trivial line bundles which correspond to $S L$-isomorphisms.

The compositions $f_{k j i}=f_{k j} \circ f_{j i} \circ f_{k i}^{-1}$ form a 2-cocycle with coefficients in $\mathbb{Z}_{2}$. Its cohomology class $w\left(\mathfrak{f}, \mathcal{E}_{i}, f_{i}\right) \in H^{2}\left(\mathcal{U}, \mathbb{Z}_{2}\right)$ is the obstruction to gluing the pairs $\left(\mathcal{E}_{i}, j_{i}\right)$ to a global family $\mathcal{E}$ on $Y \times X$ with a global isomorphism $j: \operatorname{det}(\mathcal{E}) \simeq p_{X}^{*}(\mathcal{L})$. The corresponding class $w(\mathfrak{f}) \in H^{2}\left(Y, \mathbb{Z}_{2}\right)$ depends only on $\mathfrak{f}$ and measures the obstruction to the existence of a family $\mathcal{E}$ on $Y \times X$ with an isomorphism $j: \operatorname{det}(\mathcal{E}) \simeq p_{X}^{*}(\mathcal{L})$ inducing $\mathfrak{f}$.

Since $H^{2}\left(Y, \mathcal{O}_{Y}^{*}\right)=0$, after passing to a finer cover if necessary, we can find a Cech 1-cochain $\eta=\left(\eta_{i j}\right)_{i, j} \in \check{C}^{1}\left(\mathcal{U}, \mathcal{O}_{Y}^{*}\right)$ such that $\delta(\eta)=w\left(\mathfrak{f}, \mathcal{E}_{i}, f_{i}\right)$. The system $\left(\eta_{j i} f_{j i}\right)_{j, i}$ satisfies the cocycle condition, so we can glue the bundles $\mathcal{E}_{i}$ via this system of isomorphisms and get a global bundle $\mathcal{E}$ on $Y \times X$ inducing the map $\mathfrak{f}$. The determinant of this family will be $p_{Y}^{*}(\mathcal{N}) \otimes p_{X}^{*}(\mathcal{L})$, where $\mathcal{N}$ is the line bundle associated with the cocycle $\left(\eta_{i j}^{2}\right)_{i, j}$. 
Theorem 5.2 Let $X$ be a surface of algebraic dimension $a(X)=0$, and $\mathcal{E}$ a holomorphic 2-bundle on $Y \times X$. There exists a non-empty Zariski open set $U \subset X$, a coherent sheaf $\mathcal{T}$ of rank 1 or 2 on $X$ which is locally free on $U$ and, for every $y \in Y$, a morphism $e_{y}: \mathcal{T} \rightarrow \mathcal{E}_{y}$ which is a bundle embedding (i.e. fibrewise injective) on $U$.

Proof: 3 For any holomorphic 2-bundle $\mathcal{F}$ on the curve $Y$ we put

$$
\begin{aligned}
& d(\mathcal{F}):=\min \left\{d \in \mathbb{Z} \mid \exists[\mathcal{M}] \in \operatorname{Pic}^{d}(Y) \text { s. t. } H^{0}(\mathcal{M} \otimes \mathcal{F}) \neq 0\right\}, \\
& z(\mathcal{F}):=\left\{[\mathcal{M}] \in \operatorname{Pic}^{d(\mathcal{F})}(Y) \mid H^{0}(\mathcal{M} \otimes \mathcal{F}) \neq 0\right\} \subset \operatorname{Pic}^{d(\mathcal{F})}(Y) .
\end{aligned}
$$

Note first that every $[\mathcal{M}] \in z(\mathcal{F})$ has the following two important properties

$$
\left\{\begin{array}{c}
\left.\operatorname{ker}\left[\mathrm{ev}_{y}: H^{0}(\mathcal{M} \otimes \mathcal{F}) \rightarrow \mathcal{M}(y) \otimes \mathcal{F}(y)\right)\right]=0 \forall y \in Y, \\
h^{0}(\mathcal{M} \otimes \mathcal{F}) \in\{1,2\} .
\end{array}\right.
$$

Indeed, if $s \in H^{0}(\mathcal{M} \otimes \mathcal{F}) \backslash\{0\}$ vanished at $y$, it would induce a nontrivial section in $H^{0}(\mathcal{M}(-y) \otimes \mathcal{F})$, contradicting the minimality of $d(\mathcal{F})$. The second formula follows from the first and the definition of $z(\mathcal{F})$.

For $x \in X$ denote $\mathcal{E}^{x}:=\left.\mathcal{E}\right|_{Y \times\{x\}}($ regarded as a bundle on $Y)$. The sets

$$
X_{d}:=\left\{x \in X \mid d\left(\mathcal{E}^{x}\right) \leq d\right\}
$$

are Zariski closed in $X$ and the map $x \mapsto d\left(\mathcal{E}^{x}\right)$ is bounded from above. Put

$$
\begin{gathered}
\delta:=\max _{x \in X} d\left(\mathcal{E}^{x}\right)=\min \left\{d \in \mathbb{Z} \mid \forall x \in X \exists[\mathcal{M}] \in \operatorname{Pic}^{d}(Y) \text { s. t. } H^{0}\left(\mathcal{M} \otimes \mathcal{E}^{x}\right) \neq 0\right\}, \\
W:=\left\{x \in X \mid d\left(\mathcal{E}^{x}\right)=\delta\right\} \\
Z:=\left\{(x,[\mathcal{M}]) \in X \times \operatorname{Pic}^{\delta}(Y) \mid H^{0}\left(\mathcal{M} \otimes \mathcal{E}^{x}\right) \neq 0\right\} \subset X \times \operatorname{Pic}^{\delta}(Y) .
\end{gathered}
$$

$W$ is a non-empty Zariski open set in $X$, whereas $Z$ is a closed analytic subset of $X \times \operatorname{Pic}^{\delta}(Y)$, which obviously dominates $X$. Choose any irreducible component $Z^{0}$ of $Z$ which still dominates $X$ and a non-empty Zariski open set $V \subset X$ over which the fibres $Z_{x}^{0}$ have minimal dimension. Choosing in a suitable way the multiplicities of the fibre components, one gets a holomorphic map $V \ni x \mapsto Z_{x}^{0}$ with values in the Chow variety of $\operatorname{Pic}^{\delta}(Y)([\mathrm{Ba2}$, p. 27). This map has a meromorphic extension 4 , which must be constant, because $a(X)=0$ and this Chow variety is algebraic. Let $z_{0} \subset \operatorname{Pic}^{\delta}(Y)$ be this constant, and choose $\left[\mathcal{M}_{0}\right] \in$ $z_{0}$. For $x \in V$ one has $Z_{x}^{0}=z_{0}$, whereas for $x \in W$ one has $Z_{x}^{0} \subset Z_{x}=z\left(\mathcal{E}^{x}\right)$. Therefore

$$
\left[\mathcal{M}_{0}\right] \in z\left(\mathcal{E}^{x}\right) \quad \forall x \in V \cap W .
$$

\footnotetext{
${ }^{3}$ The short proof we reproduce here was kindly suggested by one of the referees.

${ }^{4}$ The meromorphic extension theorem used here has been proved in full generality in [Ba1]; the proof is much easier when the target manifold is Kählerian (as is $\operatorname{Pic}^{\delta}(Y)$ in our case). I am indebted to D. Barlet for explaining me these results.
} 
Finally, let $U \subset V \cap W$ be the Zarisky open set of points $x \in V \cap W$ for which $h^{0}\left(\mathcal{M}_{0} \otimes \mathcal{E}^{x}\right)$ is minimal. By (12) and (13), this minimal value is either 1 or 2 . Set $\mathcal{T}:=\left(p_{X}\right)_{*}\left(p_{Y}^{*}\left(\mathcal{M}_{0}\right) \otimes \mathcal{E}\right)$. Using Grauert's local triviality and base change theorems (see [BHPV] p. 33), we see that $\mathcal{T}$ is locally free on $U$ and its fibre $\mathcal{T}(x)$ at a point $x \in U$ is $H^{0}\left(\mathcal{M}_{0} \otimes \mathcal{E}^{x}\right)$. Consider the natural morphism

$$
e: p_{X}^{*}(\mathcal{T})=p_{X}^{*}\left[\left(p_{X}\right)_{*}\left(p_{Y}^{*}\left(\mathcal{M}_{0}\right) \otimes \mathcal{E}\right)\right] \longrightarrow p_{Y}^{*}\left(\mathcal{M}_{0}\right) \otimes \mathcal{E} .
$$

For a point $(y, x) \in Y \times U$ the induced linear map $e(y, x)$ between the corresponding fibres is just the evaluation morphism

$$
H^{0}\left(\mathcal{M}_{0} \otimes \mathcal{E}^{x}\right) \longrightarrow \mathcal{M}_{0}(y) \otimes \mathcal{E}^{x}(y)=\mathcal{M}_{0}(y) \otimes \mathcal{E}_{y}(x),
$$

which is injective (by (12) and (13)). It suffices now to consider the morphisms

$$
e_{y}: \mathcal{T} \longrightarrow \mathcal{M}_{0}(y) \otimes_{\mathbb{C}} \mathcal{E}_{y} \simeq \mathcal{E}_{y}
$$

obtained by restricting $e$ to $\{y\} \times X$ and to take into account that the sheaf $p_{X}^{*}(\mathcal{T})$ is locally free on $Y \times U$.

Corollary 5.3 In the hypothesis and with the notations of Theorem 5.2 the following holds: Either $\mathcal{E}_{y}$ is filtrable for every $y \in Y$, or $\mathcal{E}_{y}$ is non-filtrable for every $y \in Y$.

Proof: When $\operatorname{rk}(\mathcal{T})=1$, all $\mathcal{E}_{y}$ will be filtrable. When $\operatorname{rk}(\mathcal{T})=2$, the bundles $\mathcal{E}_{y}$ will be either all filtrable (when $\mathcal{T}$ is filtrable), or all non-filtrable (when $\mathcal{T}$ is non-filtrable).

We can now complete the proof of our main theorem:

Proof: (of Theorem 1.2) Let $X$ be a class VII surface such that none of the rational classes $\pm c_{1}^{\mathbb{Q}}(\mathcal{K}), 0,2 c_{1}^{\mathbb{Q}}(\mathcal{K})$ is represented by a curve. Theorem 4.8 would yield a compact complex curve $Y$ and a holomorphic morphism $\mathfrak{f}: Y \rightarrow \mathcal{M}^{\text {st }}$ taking both filtrable and non-filtrable values. But a minimal class VII surface with positive $b_{2}$ has vanishing algebraic dimension. Therefore, by Lemma 5.1 and Corollary 5.3. such a morphism cannot exist.

Acknowledgments: I am grateful to Nicholas Buchdahl and Matei Toma for their encouragements and for the useful discussions we had on the preliminary version of the article. I thank the two referees for their careful and valuable suggestions. Thanks to their comments, the presentation has been improved, several errors and misprints have been fixed and the last section has been substantially simplified. I also thank G. Dloussky, K. Oeljeklaus, F. Campana and D. Barlet for their interest in my work and useful dicussions on the subject. 


\section{References}

[Ba1] Barlet, D.: Majoration du volume des fibres génériques et formes géométriques du théorème d'aplatissement, Séminaire Pierre LelongHenri Skoda (Analyse), Lecture Notes in Math. 822, 1-17 (1980).

[Ba2] Barlet, D.: How to use the cycle cpace in complex geometry Several Complex Variables MSRI Publications Vol ume 37, 25-42 (1999).

[BLP] Bănică, C.; Le Potier, J.: Sur l'existence des fibrés vectoriels holomorphes sur les surfaces non-algébriques, J. Reine Angew. Math. 378 1-31 (1987).

[BHPV] Barth, W.; Hulek, K.; Peters, Ch.; Van de Ven, A.: Compact complex surfaces, Springer (2004).

[Bo1] Bogomolov, F.: Classification of surfaces of class VII $I_{0}$ with $b_{2}=0$ Math. USSR Izv 10, 255-269 (1976).

[Bo2] Bogomolov, F.: Surfaces of class VII $I_{0}$ and affine geometry, Math. USSR Izv., 21, 31-73 (1983).

[Bu1] Buchdahl, N.: Hermitian-Einstein connections and stable vector bundles over compact complex surfaces, Math. Ann. 280, 625-648 (1988).

[Bu2] Buchdahl, N.: A Nakai-Moishezon criterion for non-Kahler surfaces, Ann. Inst. Fourier 50, 1533-1538 (2000).

[DOT] Dloussky, G.; Oeljeklaus, K.; Toma, M.: Class VII $\mathrm{V}_{0}$ surfaces with $b_{2}$ curves, Tohoku Math. J. (2) 55 no. 2, 283-309 (2003).

[Do] Donaldson, S. K.: Anti self-dual Yang-Mills connections over complex algebraic surfaces and stable vector bundles, Proc. London Math. Soc. 50, 1-26 (1985).

[DK] Donaldson, S.; Kronheimer, P.: The Geometry of Four-Manifolds, Oxford Univ. Press (1990).

[G] Gauduchon, P.: Sur la 1-forme de torsion d'une variété hermitienne compacte, Math. Ann. 267, 495-518 (1984).

[K] Kobayashi, S.: Differential geometry of complex vector bundles., Princeton Univ. Press (1987).

[LO] M. Lübke and C. Okonek: Moduli spaces of simple bundles and Hermitian-Einstein connections. Math. Ann. 276 (1987), 663-674.

[LT1] Lübke, M.; Teleman, A.: The Kobayashi-Hitchin correspondence, World Scientific Publishing Co., (1995). 
[LT2] Lübke, M.; Teleman, A.: The universal Kobayashi-Hitchin correspondence on Hermitian surfaces, math.DG/0402341, to appear in Memoirs of the AMS.

[LY] Li, J., Yau, S., T.: Hermitian Yang-Mills connections on non-Kähler manifolds, Math. aspects of string theory (San Diego, Calif., 1986), Adv. Ser. Math. Phys. 1, 560-573, World Scientific Publishing (1987).

[LYZ] Li, J.; Yau, S. T.; Zheng, F.: On projectively flat Hermitian manifolds, Comm. in Analysis and Geometry, 2, 103-109 (1994).

[Miy] Miyajima, K.: Kuranishi families of vector bundles and algebraic description of the moduli space of Einstein-Hermitian connections, Publ. R.I.M.S. Kyoto Univ. 25, 301-320 (1989).

[Na] Nakamura, I.: On surfaces of class VII $I_{0}$ with curves, Invent. Math. 78, 393-443 (1984)

[Ne] Newstead, P., E.: Introduction to moduli problems and orbit spaces, Tata Institute of Fundamental Research on Mathematics and Physics 51, Tata Institue of Fundamental Research, New-Delhi (1978)

[Te1] Teleman, A.: Projectively flat surfaces and Bogomolov's theorem on class VII $I_{0}$ - surfaces, Int. J. Math., Vol.5, No 2, 253-264 (1994)

[Te2] Teleman, A.: Instantons on class VII surfaces, in preparation.

Author's address:

Andrei Teleman, LATP, CMI, Université de Provence, 39 Rue F. Joliot-Curie, 13453 Marseille Cedex 13, France, e-mail: teleman@cmi.univ-mrs.fr. 